\title{
SOME CLASSES OF PSEUDO-BL ALGEBRAS
}

\section{GEORGE GEORGESCU and LAURENŢIU LEUŞTEAN}

(Received 7 August 2000; revised 22 August 2001)

Communicated by B. A. Davey

\begin{abstract}
Pseudo-BL algebras are noncommutative generalizations of BL-algebras and they include pseudo-MV algebras, a class of structures that are categorically equivalent to $l$-groups with strong unit. In this paper we characterize directly indecomposable pseudo-BL algebras and we define and study different classes of these structures: local, good, perfect, peculiar, and (strongly) bipartite pseudo-BL algebras.
\end{abstract}

2000 Mathematics subject classification: primary 06F99, $08 \mathrm{~A} 72$.

\section{Introduction}

BL-algebras are the algebraic structures for Hájek's Basic Logic [14]. The main example of a BL-algebra is the interval $[0,1]$ endowed with the structure induced by a t-norm. MV-algebras, Gödel algebras and product algebras are the most known classes of BL-algebras. Recent investigations are concerned with noncommutative generalizations for these structures.

In $[4,13]$, pseudo-BL algebras were defined as noncommutative generalizations of BL-algebras. The main source of examples of pseudo-BL algebras is $l$-group theory. In order to recapture some of the properties of pseudo-BL algebras a notion of pseudo- $t$-norm was introduced in [10]. For the interval $[0,1]$, this notion induces more general algebras named weak pseudo-BL algebras.

Pseudo-MV algebras were introduced as a noncommutative generalization of MValgebras (see [11, 12]). Dvurecenskij proved in [9] that the category of pseudo-MV algebras is equivalent to the category of $l$-groups with strong unit. This theorem extends the fundamental result established by Mundici for the commutative case [16].

In [2], Belluce, Di Nola and Lettieri studied local MV-algebras, structures having a unique maximal ideal. An important class of local MV-algebras are perfect MValgebras, which are MV-algebras generated by their radical. The category of perfect

(C) 2002 Australian Mathematical Society $1446-7887 / 2000 \$ A 2.00+0.00$ 
$\mathrm{MV}$-algebras is equivalent to the category of abelian l-groups [6]. All these results were extended in [15] to pseudo-MV algebras. Following [2], in [19] local BL-algebras were defined and classified.

Bipartite MV-algebras, defined in [7], are another important class of MV-algebras. Bipartite BL-algebras and strongly bipartite BL-algebras were defined in [17]. In [8] bipartite BL-algebras were classified and it was proved that the variety generated by perfect BL-algebras is exactly the variety of strongly bipartite BL-algebras. All these results are parallel to the ones already existing for MV-algebras (see [1, 7]).

In this paper we shall extend some of these results to pseudo-BL algebras. By [5], the congruences of a pseudo-BL algebra are in a bijective correspondence with the normal filters. Then, there are two possibilities to define a concept of local pseudo-BL algebra. The first one is to define a local pseudo-BL algebra as being a pseudo-BL algebra with a unique ultrafilter. This paper deals with this approach. Another way is to consider structures having a unique maximal normal filter. For the second case, we obtain the notion of normal local pseudo-BL algebra. The investigation of normal local pseudo-BL algebras seems to be a difficult problem, since we do not have a characterization of the normal filter generated by a set of elements.

The paper is divided into four sections. In the first section we recall some facts concerning pseudo-BL algebras and pseudo-MV algebras and we prove some properties used in the sequel. Following [3], we characterize directly indecomposable pseudo-BL algebras. In Section 2 we define and study local pseudo-BL algebras. Many of the results from local MV-algebras [2] and local BL-algebras [19] are extended to local pseudo-BL algebras. In the next section we study good pseudo-BL algebras, an important class of pseudo-BL algebras. We associate with any good pseudo-BL algebra a pseudo-MV algebra in a natural way. In Section 4 we investigate some classes of local pseudo-BL algebras, namely perfect, locally finite and peculiar pseudo-BL algebras. We give a classification of local pseudo-BL algebras and we give a simpler proof of the fact that locally finite pseudo-BL algebras are exactly locally finite MV-algebras. In the last section of the paper, following [17] we study (strongly) bipartite pseudo-BL algebras.

\section{Definitions and first properties}

A pseudo-BL algebra $([4,13])$ is an algebra $\mathbf{A}=(A, \wedge, \vee, \odot, \rightsquigarrow, \rightarrow, 0,1)$ with five binary operations $\wedge, \vee, \odot, \rightsquigarrow, \rightarrow$ and two constants 0,1 such that:

(A1) $(A, \wedge, \vee, 0,1)$ is a bounded lattice;

(A2) $(A, \odot, 1)$ is a monoid;

(A3) $a \odot b \leq c$ if and only if $a \leq b \leadsto c$ if and only if $b \leq a \rightarrow c$;

(A4) $a \wedge b=(a \rightsquigarrow b) \odot a=a \odot(a \rightarrow b)$;

(A5) $(a \rightsquigarrow b) \vee(b \leadsto a)=(a \rightarrow b) \vee(b \rightarrow a)=1$. 
In the sequel, we shall agree that the operations $\wedge, \vee, \odot$ have priority towards the operations $\leadsto, \rightarrow$. Sometimes, we shall put parenthesis even if this is not necessary.

It is proved in [4] that commutative pseudo-BL algebras are BL-algebras. For details on $B L-a l g e b r a s ~ s e e ~[14,18]$. A pseudo-BL algebra $A$ is nontrivial if and only if $0 \neq 1$. For any pseudo-BL algebra $A$, the reduct $L(A)=(A, \wedge, \vee, 0,1)$ is a bounded distributive lattice. A pseudo-BL chain is a linear pseudo-BL algebra, that is a pseudo-BL algebra such that its lattice order is total.

For any $a \in A$, we define $a^{\sim}=a \leadsto 0$ and $a^{-}=a \rightarrow 0$. We shall write $a^{\approx}$ instead of $\left(a^{\sim}\right)^{\sim}$ and $a^{=}$instead of $\left(a^{-}\right)^{-}$. We denote the set of natural numbers by $\omega$. We define $a^{0}=1$ and $a^{n}=a^{n-1} \odot a$ for $n \in \omega-\{0\}$. The order of $a \in A$, in symbols $\operatorname{ord}(a)$, is the smallest $n \in \omega$ such that $a^{n}=0$. If no such $n$ exists, then $\operatorname{ord}(a)=\infty$.

The following properties hold in any pseudo-BL algebra $A$ and will be used in the sequel. See [4] for details.

(1) $(a \odot b) \rightsquigarrow c=a \rightsquigarrow(b \rightsquigarrow c)$;

(2) $(b \odot a) \rightarrow c=a \rightarrow(b \rightarrow c)$;

(3) $a \leq b$ if and only if $a \leadsto b=1$ if and only if $a \rightarrow b=1$;

(4) $a \leq b$ implies $a \odot c \leq b \odot c$ and $c \odot a \leq c \odot b$;

(5) $a \odot b \leq a, b$;

(6) $a \odot b \leq a \wedge b$;

(7) $a \odot b=0$ if and only if $a \leq b^{\sim}$ if and only if $b \leq a^{-}$;

(8) $a \odot 0=0 \odot a=0$;

(9) $a^{\sim} \odot a=a \odot a^{-}=0$;

(10) $1 \rightsquigarrow a=1 \rightarrow a=a$;

(11) $a^{\sim}=1$ if and only if $a^{-}=1$ if and only if $a=0$;

(12) $1^{\sim}=1^{-}=0$;

(13) $a \leq b$ implies $b^{\sim} \leq a^{\sim}$ and $b^{-} \leq a^{-}$;

(14) $a \leq a^{--}$and $a \leq a^{-\sim}$;

(15) $a \rightsquigarrow b \leq b^{\sim} \rightarrow a^{\sim}$ and $a \rightarrow b \leq b^{-} \leadsto a^{-}$;

(16) $a^{\sim-\sim}=a^{\sim}$ and $a^{-\sim}=a^{-}$;

(17) $(a \odot b)^{\sim}=a \rightsquigarrow b^{\sim}$ and $(a \odot b)^{-}=b \rightarrow a^{-}$;

(18) $(a \vee b)^{\sim}=a^{\sim} \wedge b^{\sim}$ and $(a \vee b)^{-}=a^{-} \wedge b^{-}$;

(19) $(a \wedge b)^{\sim}=a^{\sim} \vee b^{\sim}$ and $(a \wedge b)^{-}=a^{-} \vee b^{-}$;

(20) $a \odot(b \vee c)=(a \odot b) \vee(a \odot c)$;

(21) $(b \vee c) \odot a=(b \odot a) \vee(c \odot a)$;

(22) $a \vee(b \wedge c)=(a \vee b) \wedge(a \vee c)$.

Let $\mathbf{A}$ be a pseudo-BL algebra. According to [4], a filter of $\mathbf{A}$ is a nonempty subset $F$ of $A$ such that for all $a, b \in A$,

(i) if $a, b \in F$, then $a \odot b \in F$;

(ii) if $a \in F$ and $a \leq b$, then $b \in F$. 
By (6), it is obvious that any filter of $\mathbf{A}$ is also a filter of the lattice $L(A)$. A filter $F$ of $\mathbf{A}$ is proper if $F \neq A$. A proper filter $P$ of $\mathbf{A}$ is prime if for all $a, b \in A, a \vee b \in P$ implies $a \in P$ or $b \in P$. We shall denote by $\operatorname{Spec}(A)$ the set of prime filters of the pseudo-BL algebra $A$.

A proper filter $U$ of $\mathbf{A}$ is an ultrafilter (or a maximal filter) if it is not contained in any other proper filter. We shall denote by $\mathscr{M}(A)$ the intersection of all ultrafilters of A. Obviously, $\mathscr{M}(A)$ is a proper filter of $\mathbf{A}$.

We recall some properties of filters that will be used in the sequel.

PROPOSITION 1.1 ([4, Theorem 3.25]). Let $F$ be a filter of the pseudo-BL algebra $A$ and let $S$ be $a \vee$-closed subset of $A$ (that is, if $a, b \in S$, then $a \vee b \in S$ ) such that $F \cap S=\emptyset$. Then there exists a prime filter $P$ of $\mathbf{A}$ such that $F \subseteq P$ and $P \cap S=\emptyset$.

PROPOSITION 1.2. Any proper filter of $\mathbf{A}$ can be extended to a prime filter.

PROOF. Apply [4, Corollary 3.26].

PROPOSITION 1.3 ([4, Corollary 3.32]). Any ultrafilter of $\mathbf{A}$ is a prime filter of $\mathbf{A}$.

PROPOSITION 1.4 ([4, Remark 3.33]). Any proper filter of A can be extended to an ultrafilter.

PROPOSITION 1.5. Let A be a pseudo-BL algebra. The following are equivalent:

(i) A is a pseudo-BL chain;

(ii) any proper filter of $\mathbf{A}$ is prime.

LEMMA 1.6. If $\mathrm{A}$ is a pseudo-BL algebra, then the sets $A_{0}^{\sim}=\left\{a \in A \mid a^{\sim}=0\right\}$ and $A_{0}^{-}=\left\{a \in A \mid a^{-}=0\right\}$ are proper filters of $\mathbf{A}$.

PROOF. Let us prove that $A_{0}^{\sim}$ is a proper filter of A. By (12), $1 \in A_{0}^{\sim}$. Let $a, b \in A_{0}^{\sim}$, that is, $a^{\sim}=b^{\sim}=0$. By (17), we get that $(a \odot b)^{\sim}=a \leadsto b^{\sim}=a \leadsto 0=a^{\sim}=0$, hence $a \odot b \in A_{0}^{\sim}$. Let $a \in A_{0}^{\sim}$ and $b \in A$ such that $a \leq b$. Then $a^{\sim}=0$ and, by (13), $b^{\sim} \leq a^{\sim}$, so $b^{\sim}=0$, that is, $b \in A_{0}^{\sim}$. Thus, $A_{0}^{\sim}$ is a filter of $\mathbf{A}$. Since, by (11), $0^{\sim}=1$, it follows that $0 \notin A_{0}^{\sim}$, hence $A_{0}^{\sim}$ is proper. Similarly we can show that $A_{0}^{-}$is a proper filter of $\mathbf{A}$.

Let $X \subseteq A$. The filter of $\mathbf{A}$ generated by $X$ will be denoted by $\langle X\rangle$. We have that $\langle\emptyset\rangle=\{1\}$ and $\langle X\rangle=\left\{a \in A \mid x_{1} \odot \cdots \odot x_{n} \leq a\right.$ for some $n \in \omega-\{0\}$ and some $\left.x_{1}, \ldots, x_{n} \in X\right\}$ if $\emptyset \neq X \subseteq A$. For any $a \in A,\langle a\rangle$ denotes the principal filter of $\mathbf{A}$ generated by $\{a\}$. Then, $\langle a\rangle=\left\{b \in A \mid a^{n} \leq b\right.$ for some $\left.n \in \omega-\{0\}\right\}$. 
LEMMA 1.7. Let $a, b \in A$. Then

(i) $\langle a\rangle$ is proper if and only if $\operatorname{ord}(a)=\infty$;

(ii) if $a \leq b$ and ord $(b)<\infty$, then ord $(a)<\infty$;

(iii) if $a \leq b$ and $\operatorname{ord}(a)=\infty$, then $\operatorname{ord}(b)=\infty$.

PROOF. (i) $\langle a\rangle$ is proper if and only if $0 \notin\langle a\rangle$ if and only if $a^{n} \neq 0$ for all $n \in \omega-\{0\}$ if and only if $\operatorname{ord}(a)=\infty$.

(ii), (iii) Applying (4), $a \leq b$ implies $a^{n} \leq b^{n}$ for all $n \in \omega$.

A filter $H$ of $\mathbf{A}$ is called normal ([5]) if for every $a, b \in A$ we have the equivalence:

$$
a \rightsquigarrow b \in H \quad \text { if and only if } a \rightarrow b \in H \text {. }
$$

It is easy to see that $\{1\}$ and $A$ are normal filters of the pseudo-BL algebra $\mathbf{A}$. We remark that if $\mathbf{A}$ is a BL-algebra, then $\leadsto=\rightarrow$, so the notions of filter and normal filter coincide.

For a filter $F$ of $\mathbf{A}$ and $a \in A$, let us denote $a \odot F=\{a \odot x \mid x \in F\}$ and $F \odot a=\{x \odot a \mid x \in F\}$.

PROPOSITION 1.8 ([5]). Let $H$ be a a filter of A. The following are equivalent:

(i) $H$ is a normal filter;

(ii) $a \odot H=H \odot$ a for any $a \in A$.

With any normal filter $H$ of $\mathbf{A}$ we can associate a congruence relation $\equiv_{H}$ on A by defining $a \equiv_{H} b$ if and only if $(a \leadsto b) \odot(b \leadsto a) \in H$ if and only if $(a \rightarrow b) \odot(b \rightarrow a) \in H$.

In [5] it is proved that the map $H \mapsto \equiv_{H}$ is an isomorphism between the lattice of normal filters of $\mathbf{A}$ and the lattice of congruences of $\mathbf{A}$. If we denote by $A / H$ the quotient set $A / \equiv_{\equiv_{H}}$, then $A / H$ becomes a pseudo-BL algebra $\mathrm{A} / H$ with the natural operations induced from those of $\mathbf{A}$.

PROPOSITION 1.9 ([5]). Let $H$ be a normal filter of $\mathbf{A}$. Then $\mathbf{A} / H$ is a pseudo-BL chain if and only if $H$ is a prime filter of $\mathbf{A}$.

The following lemma is implicitly contained in [5].

LEMMA 1.10. Let $H$ be a normal filter of $\mathbf{A}$ and $a, b \in A$. Then

(i) $a / H=1 / H$ if and only if $a \in H$;

(ii) $a / H=0 / H$ if and only if $a^{\sim} \in H$ if and only if $a^{-} \in H$;

(iii) $a / H \leq b / H$ if and only if $a \rightsquigarrow b \in H$ if and only if $a \rightarrow b \in H$. 
PROOF. (i) $a / H=1 / H$ if and only if $(a \rightsquigarrow 1) \odot(1 \rightsquigarrow a) \in H$ if and only if $1 \odot(1 \leadsto a) \in H$ if and only if $a \in H$, since $a \rightsquigarrow 1=1$ and $1 \leadsto a=a$, by (3) and (10).

(ii) $a / H=0 / H$ if and only if $(a \leadsto 0) \odot(0 \leadsto a) \in H$ if and only if $a^{\sim} \odot 1 \in H$ if and only if $a^{\sim} \in H$. Applying $((N)), a^{\sim} \in H$ if and only if $a \rightsquigarrow 0 \in H$ if and only if $a \rightarrow 0 \in H$ if and only if $a^{-} \in H$.

(iii) By (3) and (i), $a / H \leq b / H$ if and only if $a / H \leadsto b / H=1 / H$ if and only if $(a \rightsquigarrow b) / H=1 / H$ if and only if $a \rightsquigarrow b \in H$. By (N), we have that $a \rightsquigarrow b \in H$ if and only if $a \rightarrow b \in H$.

If $h: \mathbf{A} \rightarrow \mathbf{B}$ is a homomorphism of pseudo-BL algebras, then the kernel of $h$ is the set $\operatorname{Ker}(h)=\{a \in A \mid h(a)=1\}$. For any normal filter $H$ of $\mathbf{A}$, let us denote by []$_{H}$ the natural homomorphism from $\mathbf{A}$ onto $\mathrm{A} / H$, defined by []$_{H}(a)=a / H$ for any $a \in A$. Then $H=\operatorname{Ker}\left([]_{H}\right)$. The following propositions are easily obtained.

PROPOSITION 1.11. Let $h: \mathrm{A} \rightarrow \mathrm{B}$ be a homomorphism of pseudo-BL algebras. Then the following properties hold:

(i) for any (normal) filter $G$ of $\mathbf{B}$, the set $h^{-1}(G)=_{\text {def }}\{a \in A \mid h(a) \in G\}$ is a (normal) filter of $\mathbf{A}$. Thus, in particular $\operatorname{Ker}(h)$ is a normal filter of $\mathbf{A}$.

(ii) $h$ is injective if and only if $\operatorname{Ker}(h)=\{1\}$.

PROPOSITION 1.12. Let $\mathrm{A}$ be a pseudo-BL algebra and $H$ be a normal filter of $\mathbf{A}$.

(i) The map $F \stackrel{\alpha}{\mapsto}[]_{H}(F)$ is an inclusion-preserving bijective correspondence between the filters of $\mathbf{A}$ containing $H$ and the filters of $\mathbf{A} / H$. The inverse map is also inclusion-preserving.

(ii) $F$ is a proper filter of $\mathbf{A}$ containing $H$ if and only if []$_{H}(F)$ is a proper filter of $\mathbf{A} / H$. Hence, there is a bijection between the proper filters of $\mathbf{A}$ containing $H$ and the proper filters of $\mathbf{A} / H$.

(iii) There is a bijection between the ultrafilters of $\mathbf{A}$ containing $H$ and the ultrafilters of $\mathbf{A} / H$.

Let $\mathbf{A}$ be a pseudo-BL algebra and $F$ be a filter of $\mathbf{A}$. We shall use the following notation:

$$
\begin{aligned}
& F_{\sim}^{*}=\left\{a \in A \mid a \leq x^{\sim} \text { for some } x \in F\right\} \text { and } \\
& F_{-}^{*}=\left\{a \in A \mid a \leq x^{-} \text {for some } x \in F\right\} .
\end{aligned}
$$

REMARK 1.13. Let $\mathbf{A}$ be a pseudo-BL algebra. Then

(i) $F_{\sim}^{*}=\{a \in A \mid a \odot x=0$ for some $x \in F\}$;

(i') $F_{-}^{*}=\{a \in A \mid x \odot a=0$ for some $x \in F\}$; 
(ii) $F_{\sim}^{*}=\left\{a \in A \mid a^{-} \in F\right\}$;

(ii') $F_{-}^{*}=\left\{a \in A \mid a^{\sim} \in F\right\}$.

PROOF. (i), (i') Apply (7).

(ii) Let $a \in A$. If $a \leq x^{\sim}$ for some $x \in F$ then, by (13) and (14), we get that $x \leq x^{\sim-} \leq a^{-}$. Since $F$ is a filter, it follows that $a^{-} \in F$. Conversely, suppose that $a^{-} \in F$. Then, $a \leq\left(a^{-}\right)^{\sim}$, hence $a \in F_{\sim}^{*}$.

(ii') Similar to (ii).

For any pseudo-BL algebra $\mathrm{A}, B(A)$ denotes the Boolean algebra of all complemented elements in $L(A)$. Hence, $B(A)=B(L(A))$.

PROPOSITION 1.14 ([5]). Let $\mathbf{A}$ be a pseudo-BL algebra and $e \in A$. The following are equivalent:

(i) $e \in B(A)$;

(ii) $e \odot e=e$ and $e=e^{\sim-}=e^{-\sim}$;

(iii) $e \odot e=e$ and $e^{\sim} \leadsto e=e$;

(iii') $e \odot e=e$ and $e^{-} \rightarrow e=e$;

(iv) $e \vee e^{\sim}=1$;

(iv') $e \vee e^{-}=1$.

LEMMA 1.15 ([5]). Let $\mathrm{A}$ be a pseudo-BL algebra and $e \in B(A)$. Then

(i) $\langle e\rangle=\{a \in A \mid e \leq a\}$;

(ii) $e \odot a=e \wedge$ a for any $a \in A$;

(iii) $e \vee(a \odot b)=(e \vee a) \odot(e \vee b)$ for any $a, b \in A$;

(iv) $e^{\sim}=e^{-}$is the complement of $e$.

A pseudo-BL algebra $\mathbf{A}$ is called directly indecomposable if and only if $\mathbf{A}$ is nontrivial and whenever $\mathbf{A} \cong \mathbf{A}_{1} \times \mathbf{A}_{2}$ then either $\mathbf{A}_{1}$ or $\mathbf{A}_{2}$ is trivial. In the sequel, in a similar manner as in [3, Chapter 6.4], we shall give a characterization of directly indecomposable pseudo-BL algebras. Let $\mathrm{A}$ be a pseudo-BL algebra. For each $x \in A$, let the functions $\rightsquigarrow_{x}: A \times A \rightarrow A, \rightarrow_{x}: A \times A \rightarrow A$ and $h_{x}: A \rightarrow A$ be defined by $a \rightsquigarrow_{x} b=x \vee(a \rightsquigarrow b), a \rightarrow_{x} b=x \vee(a \rightarrow b)$, and $h_{x}(a)=x \vee a$.

Proposition 1.16. Let $\mathrm{A}$ be a pseudo-BL algebra and $e \in B(A)$. Then

(i) $\langle\mathbf{e}\rangle=\left(\langle e\rangle, \wedge, \vee, \odot, \rightsquigarrow_{e}, \rightarrow_{e}, e, 1\right)$ is a pseudo-BL algebra;

(ii) $h_{e}(A)=\langle e\rangle$;

(iii) $h_{e}$ is a homomorphism of pseudo-BL algebras from $\mathbf{A}$ onto $\langle\mathbf{e}\rangle$;

(iv) $\operatorname{Ker}\left(h_{e}\right)=\left\langle e^{-}\right\rangle$;

(v) $\langle\mathbf{e}\rangle$ is nontrivial if and only if $e \neq 1$;

(vi) $\langle e\rangle$ is a subalgebra of $\mathbf{A}$ if and only if $e=0$ if and only if $\langle e\rangle=A$; 
(vii) $B(\langle e\rangle)=\langle e\rangle \cap B(A)$.

ProOF. (i) By Lemma 1.15 (i), we have that $\langle e\rangle=\{a \in A \mid e \leq a\}$. Let us verify the axioms from the definition of a pseudo-BL algebra.

(A1) It follows immediately that $(\langle e\rangle, \wedge, \vee, e, 1)$ is a bounded lattice.

(A2) Since $\langle e\rangle$ is a filter of $\mathbf{A},\langle e\rangle$ is $\odot$-closed and, obviously, $(\langle e\rangle, \odot, 1)$ is a monoid.

(A3) Let $a, b, c \geq e$. If $a \odot b \leq c$, then $a \leq b \leadsto c \leq e \vee(b \rightsquigarrow c)=b \rightsquigarrow_{e} c$ and $b \leq a \rightarrow c \leq e \vee(a \rightarrow c)=a \rightarrow_{e} c$.

Conversely, let us suppose that $a \leq b \rightsquigarrow_{e} c$, that is, $a \leq e \vee(b \leadsto c)$. Applying (4), (21), Lemma 1.15 (ii) and (A4), we get that $a \odot b \leq[e \vee(b \leadsto c)] \odot b=$ $(e \odot b) \vee[(b \leadsto c) \odot b]=(e \wedge b) \vee(b \wedge c)=e \vee(b \wedge c)=b \wedge c \leq c$.

Now, let us suppose that $b \leq a \rightarrow_{e} c$, so $b \leq e \vee(a \rightarrow c)$. Then, by (4), (20), Lemma 1.15 (ii) and (A4), $a \odot b \leq a \odot[e \vee(a \rightarrow c)]=(a \odot e) \vee[a \odot(a \rightarrow c)]=$ $(a \wedge e) \vee(a \wedge c)=e \vee(a \wedge c)=a \wedge c \leq c$.

(A4) Let $a, b \geq e$. We have that $\left(a \rightsquigarrow_{e} b\right) \odot a=[e \vee(a \rightsquigarrow b)] \odot a=$ $(e \odot a) \vee[(a \leadsto b) \odot a]=(e \wedge a) \vee(a \wedge b)=e \vee(a \wedge b)=a \wedge b$ and, similarly, $a \odot\left(a \rightarrow_{e} b\right)=a \odot[e \vee(a \rightarrow b)]=(a \odot e) \vee[a \odot(a \rightarrow b)]=(a \wedge e) \vee(a \wedge b)=a \wedge b$.

(A5) Let $a, b \in A$. By (A5), we get that $\left(a \rightsquigarrow_{e} b\right) \vee\left(b \rightsquigarrow_{e} a\right)=e \vee(a \rightsquigarrow$ b) $\vee e \vee(b \rightsquigarrow a)=e \vee 1=1$ and, similarly, $\left(a \rightarrow_{e} b\right) \vee\left(b \rightarrow_{e} a\right)=e \vee(a \rightarrow$ b) $\vee e \vee(b \rightarrow a)=e \vee 1=1$. Hence, $\left(\langle e\rangle, \wedge, \vee, \odot, \sim_{e}, \rightarrow_{e}, e, 1\right)$ is a pseudo-BL algebra.

(ii) For any $a \in\langle e\rangle$, we have that $h_{e}(a)=e \vee a=a$. Hence, $\langle e\rangle \subseteq h_{e}(A)$. The other inclusion is obvious.

(iii) Let $a, b \in A$. It follows immediately that $h_{e}(a \rightsquigarrow b)=e \vee(a \rightsquigarrow b)=a \rightsquigarrow_{e} b$, $h_{e}(a \rightarrow b)=e \vee(a \rightarrow b)=a \rightarrow_{e} b, h_{e}(0)=0 \vee e=e, h_{e}(1)=e \vee 1=1$, $h_{e}(a \vee b)=e \vee(a \vee b)=h_{e}(a) \vee h_{e}(b)$. By (22), $h_{e}(a \wedge b)=e \vee(a \wedge b)=$ $(e \vee a) \wedge(e \vee b)=h_{e}(a) \wedge h_{e}(b)$. Applying Lemma 1.15 (iii), we also get that $h_{e}(a \odot b)=e \vee(a \odot b)=(e \vee a) \odot(e \vee b)=h_{e}(a) \odot h_{e}(b)$.

(iv) If $a \in \operatorname{Ker}\left(h_{e}\right)$, then $h_{e}(a)=a \vee e=1$, so $e^{-}=e^{-} \wedge(a \vee e)=\left(e^{-} \wedge a\right) \vee 0=$ $e^{-} \wedge a$. It follows that $a \geq e^{-}$, hence $a \in\left\langle e^{-}\right\rangle$. Conversely, if $a \geq e^{-}$, we get that $h_{e}(a)=e \vee a \geq e \vee e^{-}=1$, hence $h_{e}(a)=1$, that is, $a \in \operatorname{Ker}\left(h_{e}\right)$.

(v), (vi) They are obvious.

(vii) Let $a \in\langle e\rangle$, that is, $e \leq a$. If $a \in B(\langle e\rangle)$, then there is $b \geq e$ such that $a \wedge b=e$ and $a \vee b=1$. Taking $c=b \wedge e^{\sim}$, we get that $a \wedge c=0$ and $a \vee c=a \vee\left(b \wedge e^{\sim}\right)=(a \vee b) \wedge\left(a \vee e^{\sim}\right)=1 \wedge\left(a \vee e^{\sim}\right)=a \vee e^{\sim} \geq e \vee e^{\sim}=1$, by (22) and Lemma 1.15 (iv). Conversely, suppose that $a \in B(A)$, hence there is $b \in A$ such that $a \vee b=1$ and $a \wedge b=0$. Let $c=e \vee b$. Then $c \geq e$ and $a \vee c=1$, $a \wedge c=a \wedge(e \vee b)=(a \wedge e) \vee(a \wedge b)=e \vee 0=e$. 
PROPOSITION 1.17. Let $\left\{\mathbf{A}_{i}\right\}_{i \in I}$ be a nonempty family of pseudo-BL algebras and let $\mathbf{P}=\prod_{i \in I} \mathbf{A}_{i}$. Then there exists a set $\left\{\delta_{i} \mid i \in I\right\} \subseteq B(P)$ satisfying the following conditions:

(i) $\wedge_{i \in l} \delta_{i}=0$;

(ii) $\delta_{i} \vee \delta_{j}=1$, whenever $i \neq j$;

(iii) each $\mathbf{A}_{i}$ is isomorphic to $\left\langle\boldsymbol{\delta}_{i}\right\rangle$.

PROOF. Similar to the proof of [3, Lemma 6.4.4].

PROPOSITION 1.18. Let $\mathbf{A}$ be a pseudo-BL algebra and $e_{1}, \ldots, e_{n} \in B(A), n \geq 2$, such that

(i) $e_{1} \wedge \cdots \wedge e_{n}=0$; and

(ii) $e_{i} \vee e_{j}=1$ for $i \neq j, i, j=1, \ldots, n$.

Then $\mathbf{A} \cong\left\langle\mathbf{e}_{1}\right\rangle \times \cdots \times\left\langle\mathbf{e}_{n}\right\rangle$.

PROOF. Similar to the proof of [3, Lemma 6.4.5].

PROPOSITION 1.19. A pseudo-BL algebra $\mathbf{A}$ is directly indecomposable if and only if $B(A)=\{0,1\}$.

PROOF. Similar to the proof of [3, Theorem 6.4.7].

It follows immediately that

PROPOSITION 1.20. Any pseudo-BL chain is directly indecomposable.

Proof. Let $\mathrm{A}$ be a pseudo-BL chain and $e \in B(A)$. By Proposition 1.14, we get that $e \vee e^{\sim}=1$. But $e \leq e^{\sim}$ or $e^{\sim} \leq e$, hence $e=1$ or $e^{\sim}=1$. By (11), it follows that $e \in\{0,1\}$.

In the sequel we shall recall some facts about pseudo-MV algebras, which are noncommutative generalizations of MV-algebras (see [11, 12]). A pseudo-MV algebra is an algebra $\left(A, \oplus,^{-}, \sim, 0,1\right)$ with one binary operation $\oplus$, two unary operations ${ }^{-}, \sim$ and two constants 0,1 such that:

(i) $(A, \oplus, 0)$ is a monoid;

(ii) $a \oplus 1=1 \oplus a=a$;

(iii) $1^{\sim}=1^{-}=0$;

(iv) $\left(a^{-} \oplus b^{-}\right)^{\sim}=\left(a^{\sim} \oplus b^{\sim}\right)^{-}$;

(v) $a \oplus\left(a^{\sim} \odot b\right)=b \oplus\left(b^{\sim} \odot a\right)=\left(a \odot b^{-}\right) \oplus b=\left(b \odot a^{-}\right) \oplus a$;

(vi) $a \odot\left(a^{-} \oplus b\right)=\left(a \oplus b^{\sim}\right) \odot b$;

(vii) $a^{-\sim}=a$, 
where $a \odot b \stackrel{\text { def }}{=}\left(b^{-} \oplus a^{-}\right)^{\sim}$. Let $\mathbf{A}$ be a pseudo-MV algebra. On $A$ one can define an order relation ' $\leq$ ' by

$$
a \leq b \text { if and only if } a^{-} \oplus b=1 \text { if and only if } b \oplus a^{\sim}=1
$$

Proposition 1.21 ([11, Proposition 1.13]). Let A be a pseudo-MV algebra. Then $(A, \leq)$ is a lattice in which for all $a, b \in A$,

$a \vee b=a \oplus\left(a^{\sim} \odot b\right)=b \oplus\left(b^{\sim} \odot a\right)=\left(a \odot b^{-}\right) \oplus b=\left(b \odot a^{-}\right) \oplus a \quad$ and $a \wedge b=\left(a \oplus b^{\sim}\right) \odot b=\left(b \oplus a^{\sim}\right) \odot a=a \odot\left(a^{-} \oplus b\right)=b \odot\left(b^{-} \oplus a\right)$.

For any $a \in A$, we define $0 a=0$ and $n a=(n-1) a \oplus a$ for $n \in \omega-\{0\}$. The MV-order of $a \in A$, in symbols MV-ord $(a)$, is the smallest $n \in \omega$ such that $n a=1$. If no such $n$ exists, then MV-ord $(a)=\infty$.

LEMMA 1.22 ([15, Lemma 14]). Let A be a pseudo-MV algebra. For any $a \in A$, $\operatorname{MV}$-ord $\left(a^{-}\right)=\mathrm{MV}$-ord $\left(a^{\sim}\right)$.

We shall denote by $D(A)$ the set $\{a \in A \mid \mathrm{MV}$-ord $(a)=\infty\}$. A pseudo-MV algebra $\mathbf{A}$ is locally finite if for all $a \in A, a \neq 0$ implies MV-ord $(a)<\infty$. According to [15], a pseudo-MV algebra $A$ is strong if for all $a \in A, a^{-}=a^{\sim}$. According to [11], an ideal of $\mathbf{A}$ is is a nonempty subset $I$ of $A$ such that for all $a, b \in A$,

(i) if $a, b \in I$, then $a \oplus b \in I$;

(ii) if $b \in I$ and $a \leq b$, then $a \in I$.

An ideal $I$ is proper if $I \neq A$. A proper ideal of $\mathbf{A}$ is called a maximal ideal if it is not contained in any other proper ideal. An ideal $H$ of a pseudo-MV algebra $A$ is called normal (see [12]) if for all $a, b \in A, a^{\sim} \odot b \in H$ if and only if $b \odot a^{-} \in H$.

LEMMA 1.23 ([12, Lemma 3.2]). Let $H$ be a normal ideal of $\mathbf{A}$ and $a \in A$. Then $a \in H$ if and only if $a^{=} \in H$ if and only if $a^{\approx} \in H$.

Proposition 1.24 ([4, Corollary 2.34]). A pseudo-BL algebra $\mathbf{A}$ is a pseudo-MV algebra if and only if $a^{\sim-}=a^{-\sim}=a$ for all $a \in A$.

Following [2], in [15] local pseudo-MV algebras were defined and some classes of local pseudo-MV algebras were studied. Thus, a pseudo-MV algebra is local if and only if it has a unique maximal ideal and a local pseudo-MV algebra is:

- perfect if for any $a \in A, \mathrm{MV}$-ord $(a)<\infty$ if and only if MV-ord $\left(a^{-}\right)=\infty$;

- singular if there exist $a, b \in A$ such that $\mathrm{MV}$-ord $(a)<\infty, \mathrm{MV}$-ord $(b)<\infty$ and MV-ord $(a \odot b)=\infty$. 
By Lemma 1.22, it follows that a local pseudo-MV algebra $\mathbf{A}$ is perfect if and only if for any $a \in A, M V-o r d(a)<\infty$ if and only if $M V-\operatorname{ord}\left(a^{\sim}\right)=\infty$

PROPOSITION 1.25 ([15]). Every local pseudo-MV algebra is either perfect or singular. There is no local pseudo-MV algebra which is both perfect and singular.

PROPOSITION 1.26 ([15]). Every locally finite pseudo-MV algebra different from $\{0,1\}$ is singular.

\section{Local pseudo-BL algebras}

Local rings play an important role in ring theory. On the other hand, the study of local objects became a standard problem for other classes of structures (MV-algebras [2], BL-algebras [19], pseudo-MV algebras [15]). In this section we shall study local pseudo-BL algebras.

A pseudo-BL algebra is called local if and only if it has a unique ultrafilter.

LEMMA 2.1. Let A be a local pseudo-BL algebra. Then

(i) any proper filter of $\mathbf{A}$ is included in the unique ultrafilter of $\mathbf{A}$;

(ii) $A_{0}^{\sim}, A_{0}^{-}$are included in the unique ultrafilter of $\mathbf{A}$.

Proof. (i) Apply Proposition 1.4 and the fact that $\mathbf{A}$ has a unique ultrafilter.

(ii) Apply Lemma 1.6 and (i).

In the sequel, we shall use the following notation:

$$
D(A)=\{a \in A \mid \operatorname{ord}(a)=\infty\} \quad \text { and } D(A)^{*}=\{a \in A \mid \operatorname{ord}(a)<\infty\} .
$$

Obviously, $D(A) \cap D(A)^{*}=\emptyset$ and $D(A) \cup D(A)^{*}=A$.

Proposition 2.2. Let $\mathrm{A}$ be a pseudo-BL algebra. The following are equivalent:

(i) $D(A)$ is a filter of $\mathbf{A}$;

(ii) $D(A)$ is a proper filter of $\mathbf{A}$;

(iii) $\mathbf{A}$ is local;

(iv) $D(A)$ is the unique ultrafilter of $\mathbf{A}$;

(v) for all $a, b \in A$, ord $(a \odot b)<\infty$ implies $\operatorname{ord}(a)<\infty$ or $\operatorname{ord}(b)<\infty$.

PROOF. (i) if and only if (ii). We have that ord(0)=1, hence $0 \notin D(A)$. 
(i) implies (v). Let $a, b \in A$ such that $\operatorname{ord}(a \odot b)<\infty$, so $a \odot b \notin D(A)$. Since $D(A)$ is a filter of $\mathbf{A}$, we get that $a \notin D(A)$ or $b \notin D(A)$. Hence, ord $(a)<\infty$ or $\operatorname{ord}(b)<\infty$.

(v) implies (i). Since $1 \in D(A)$, we have that $D(A)$ is nonempty. Let $a, b \in D(A)$, that is $\operatorname{ord}(a)=\operatorname{ord}(b)=\infty$. It follows that $\operatorname{ord}(a \odot b)=\infty$, that is $a \odot b \in D(A)$. If $a \leq b$ and $a \in D(A)$, then $a^{n}>0$ for all $n \in \omega$. Since $a^{n} \leq b^{n}$, we have that $b^{n}>0$ for all $n \in \omega$. That is, ord $(b)=\infty$, hence $b \in D(A)$. Thus, we have proved that $D(A)$ is a filter of $\mathbf{A}$.

(iv) implies (iii). It is immediate.

(iii) implies (iv). Let $U$ be the unique ultrafilter of A. Applying Lemma 2.1 (i) and Lemma 1.7 (i), we get that $a \in U$ if and only if $\langle a\rangle \subseteq U$ if and only if $\langle a\rangle$ is proper if and only if $\operatorname{ord}(a)=\infty$ if and only if $a \in D(A)$. Hence, $U=D(A)$.

(iv) implies (i). It is obvious.

(i) implies (iv). Since $0 \notin D(A)$, we have that $D(A)$ is proper. Let $F$ be a proper filter of A. If $a \in F$, then $\langle a\rangle \subseteq F$, so $\langle a\rangle$ is a proper filter of A. Applying Lemma 1.7 (i), it follows that ord $(a)=\infty$, hence $a \in D(A)$. Thus, we have got that any proper filter $F$ of $\mathbf{A}$ is included in $D(A)$. From this fact it follows that $D(A)$ is the unique ultrafilter of $\mathbf{A}$.

COROLLARY 2.3. Let A be a local pseudo-BL algebra. Then

(i) for any $a \in A$, ord $(a)<\infty$ or $\left(\operatorname{ord}\left(a^{\sim}\right)<\infty\right.$ and $\left.\operatorname{ord}\left(a^{-}\right)<\infty\right)$;

(ii) $D(A)_{\sim}^{*} \subseteq D(A)^{*}$ and $D(A)_{-}^{*} \subseteq D(A)^{*}$;

(iii) $D(A) \cap D(A)_{\sim}^{*}=D(A) \cap D(A)_{-}^{*}=\emptyset$.

PROOF. (i) Let $a \in A$. By (9), we have that $a^{\sim} \odot a=a \odot a^{-}=0$, so ord $\left(a^{\sim} \odot a\right)=$ $\operatorname{ord}\left(a \odot a^{-}\right)=\operatorname{ord}(0)=1<\infty$. Apply now Proposition 2.2 (v) to get (i).

(ii) Let $a \in D(A)_{\sim}^{*}$, so there is $x \in D(A)$ such that $a \leq x^{\sim}$. Since ord $(x)=\infty$, applying (i), we get that ord $\left(x^{\sim}\right)<\infty$. Applying now Lemma 1.7(ii), we get that ord $(a)<\infty$. Hence, $a \in D(A)^{*}$. We obtain similarly that $D(A)_{-}^{*} \subseteq D(A)^{*}$.

(iii) Apply (ii) and the fact that $D(A) \cap D(A)^{*}=\emptyset$.

PROPOSITION 2.4. Any pseudo-BL chain is a local pseudo-BL algebra.

Proof. Let $\mathbf{A}$ be a pseudo-BL chain. We apply Proposition 2.2 (v) to obtain that $\mathbf{A}$ is local. Let $a, b \in A$ such that $\operatorname{ord}(a \odot b)<\infty$. Since $\mathbf{A}$ is a chain, we have that $a \leq b$ or $b \leq a$. Suppose that $a \leq b$. Then $a \odot a \leq a \odot b$, so, by Lemma 1.7 (ii), we get that ord $(a \odot a)<\infty$, hence ord $(a)<\infty$. Similarly, from $b \leq a$ it follows that that $\operatorname{ord}(b)<\infty$.

A proper normal filter $P$ of a pseudo-BL algebra $\mathbf{A}$ is called primary if for all 
$a, b \in A$,

$\left((a \odot b)^{n}\right)^{\sim} \in P$ for some $n \in \omega$ implies $\left(a^{m}\right)^{\sim} \in P$ or $\left(b^{m}\right)^{\sim} \in P$ for some $m \in \omega$.

Applying the definition of a normal filter, we get that a proper normal filter $P$ of $\mathbf{A}$ is primary if and only if for all $a, b \in A,\left((a \odot b)^{n}\right)^{-} \in P$ for some $n \in \omega$ implies $\left(a^{m}\right)^{-} \in P$ or $\left(b^{m}\right)^{-} \in P$ for some $m \in \omega$.

REMARK 2.5. Suppose that $\mathbf{A}$ is a BL-algebra and let $P$ be a proper filter of $\mathbf{A}$. The following are equivalent:

(i) $P$ is primary;

(ii) for all $a, b \in A,(a \odot b)^{-} \in P$ implies $\left(a^{m}\right)^{-} \in P$ or $\left(b^{m}\right)^{-} \in P$ for some $m \in \omega$.

PROOF. (i) implies (ii). It follows immediately from the definition of a primary filter.

(ii) implies (i). Let $a, b \in A$ such that $\left((a \odot b)^{n}\right)^{-} \in P$ for some $n \in \omega$. Since $\odot$ is commutative, we get that $\left((a \odot b)^{n}\right)^{-}=\left(a^{n} \odot b^{n}\right)^{-} \in P$. Applying now (ii), it follows that there is $p \in \omega$ such that $\left(a^{n p}\right)^{-} \in P$ or $\left(b^{n p}\right)^{-} \in P$. Hence, letting $m=n p$, we have that $\left(a^{m}\right)^{-} \in P$ or $\left(b^{m}\right)^{-} \in P$.

Hence, in the case that $\mathbf{A}$ is a BL-algebra, the notion of primary filter defined here coincides with the notion of primary filter defined in [19].

PROPOSITION 2.6. Let $\mathbf{A}$ be a pseudo-BL algebra and $P$ be a proper normal filter of A. The following are equivalent:

(i) $\mathbf{A} / P$ is a local pseudo-BL algebra;

(ii) $P$ is a primary filter of $\mathbf{A}$.

Proof. Applying Proposition 2.2 (v) and Lemma 1.10 (ii), we have that $\mathbf{A} / P$ is local if and only if for all $a, b \in A$, ord $(a / P \odot b / P)<\infty$ implies ord $(a / P)<\infty$ or $\operatorname{ord}(b / P)<\infty$ if and only if for all $a, b \in A,(a / P \odot b / P)^{n}=0 / P$ for some $n \in \omega$ implies $(a / P)^{m}=0 / P$ or $(b / P)^{m}=0 / P$ for some $m \in \omega$ if and only if for all $a, b \in A,\left((a \odot b)^{n}\right) / P=0 / P$ for some $n \in \omega$ implies $a^{m} / P=0 / P$ or $b^{m} / P=0 / P$ for some $m \in \omega$ if and only if for all $a, b \in A,\left((a \odot b)^{n}\right)^{\sim} \in P$ for some $n \in \omega$ implies $\left(a^{m}\right)^{\sim} \in P$ or $\left(b^{m}\right)^{\sim} \in P$ for some $m \in \omega$ if and only if $P$ is primary.

PROPOSITION 2.7. Any prime normal filter of a pseudo-BL algebra $\mathbf{A}$ is primary.

ProOF. Let $P$ be a prime normal filter of A. Applying Proposition 1.9, we get that $\mathbf{A} / P$ is a pseudo-BL chain, hence $\mathbf{A} / P$ is local, by Proposition 2.4. Apply now Proposition 2.6 to get that $P$ is primary. 
PROPOSITION 2.8. Let $\mathrm{A}$ be a pseudo-BL algebra. A proper normal filter of $\mathrm{A}$ is primary if and only if it is contained in a unique ultrafilter of $\mathbf{A}$.

PROOF. Let $H$ be a proper normal filter of A. By Proposition 2.6, $H$ is primary if and only if $\mathbf{A} / H$ is a local algebra if and only if $\mathbf{A} / H$ has a unique ultrafilter. Applying Proposition 1.12 (iii), there is a bijection between the set of ultrafilters of $\mathbf{A} / H$ and the set of ultrafilters of $\mathbf{A}$ that contain $H$. Hence, $H$ is primary if and only if there is a unique ultrafilter of $\mathbf{A}$ that contains $H$.

PROPOSITION 2.9. Let $\mathrm{A}$ be a pseudo-BL algebra. The following are equivalent:

(i) A is local;

(ii) any proper normal filter of $\mathrm{A}$ is primary;

(iii) $\{1\}$ is a primary filter of $\mathbf{A}$.

ProOF. (i) implies (ii). Let $H$ be a proper normal filter of $\mathbf{A}$. Since $\mathbf{A}$ is local, by Lemma 2.1 (i) and Proposition 2.2 (iv) it follows that $D(A)$ is the unique ultrafilter of A containing $H$. Applying Proposition 2.8, we get that $H$ is primary.

(ii) implies (iii). Apply the fact that $\{1\}$ is a proper normal filter of $\mathbf{A}$.

(iii) implies (i). Since $\{1\}$ is a primary filter of $\mathbf{A}$, by Proposition 2.6 , we get that $\mathbf{A} /\{1\}$ is local. But $\mathbf{A} \cong \mathbf{A} /\{1\}$, hence $\mathbf{A}$ is local.

PROPOSITION 2.10. Any local pseudo-BL algebra is directly indecomposable.

PROOF. Let $\mathrm{A}$ be a local pseudo-BL algebra. We shall prove that $B(A)=\{0,1\}$ and then apply Proposition 1.19. Let $e \in B(A)$. Applying Corollary 2.3 (i), we get that $\operatorname{ord}(e)<\infty$ or $\operatorname{ord}\left(e^{\sim}\right)<\infty$, that is, there is $n \in \omega-\{0\}$ such that $e^{n}=0$ or $\left(e^{\sim}\right)^{n}=0$. But $e^{n}=e$ and $\left(e^{\sim}\right)^{n}=e^{\sim}$, by Proposition 1.14 (ii) and the fact that $e^{\sim}$ is the complement of $e$, so $e, e^{\sim} \in B(A)$. It follows that $e=0$ or $e^{\sim}=0$. By Proposition 1.14 (ii) and (11), from $e^{\sim}=0$ we get that $e=\left(e^{\sim}\right)^{-}=0^{-}=1$. That is, $e \in\{0,1\}$. Hence, $B(A)=\{0,1\}$.

\section{Good pseudo-BL algebras}

A good pseudo-BL algebra is a pseudo-BL algebra A satisfying the following identity

$$
a^{\sim-}=a^{-\sim} \text {. }
$$

Pseudo-MV algebras are particular cases of good pseudo-BL algebras. In [5] it is proved that any pseudo-product algebra is also a good pseudo-BL algebra. A strong 
pseudo-BL algebra is a pseudo-BL algebra $\mathbf{A}$ such that $a^{\sim}=a^{-}$for all $a \in A$. Obviously, every strong pseudo-BL algebra is a good pseudo-BL algebra.

In the sequel, if not otherwise specified, $\mathbf{A}$ is a good pseudo-BL algebra. Let us consider the subset $M(A)=\left\{a \in A \mid a^{\sim-}=a^{-\sim}=a\right\}$.

LEMMA 3.1. Let $\mathbf{A}$ be a good pseudo-BL algebra. Then

(i) $0,1 \in M(A)$;

(ii) $a^{\sim}, a^{-} \in M(A)$ for all $a \in A$;

(iii) if $a, b \in M(A)$, then $a \rightsquigarrow b=b^{\sim} \rightarrow a^{\sim}$ and $a \rightarrow b=b^{-} \rightsquigarrow a^{-}$;

(iv) if $a, b \in M(A)$, then $\left(a^{\sim} \odot b^{\sim}\right)^{-}=\left(a^{-} \odot b^{-}\right)^{\sim}=a^{-} \leadsto b=b^{\sim} \rightarrow a$.

ProOF. (i) Apply (11) and (12).

(ii) Let $a \in A$. Applying (*) for $a^{\sim}$ and $a^{-}$and (16), we have that $\left(a^{\sim}\right)^{\sim-}=$ $\left(a^{\sim}\right)^{-\sim}=a^{\sim}$ and $\left(a^{-}\right)^{-\sim}=\left(a^{-}\right)^{\sim-}=a^{-}$. It follows that $a^{\sim}, a^{-} \in M(A)$.

(iii), (iv) See [4, Lemma 2.31].

For any $a, b \in A$, let us define $a \oplus b \stackrel{\text { def }}{=}\left(b^{\sim} \odot a^{\sim}\right)^{-}$.

LEMMA 3.2. Let $\mathbf{A}$ be a good pseudo-BL algebra. Then

(i) $a \oplus b \in M(A)$ for any $a, b \in A$;

(ii) if $a, b \in M(A)$, then $a \oplus b=\left(b^{\sim} \odot a^{\sim}\right)^{-}=\left(b^{-} \odot a^{-}\right)^{\sim}=b^{-} \rightsquigarrow a=a^{\sim} \rightarrow b$;

(iii) if $a, b \in M(A)$, then $a \oplus b^{-}=a^{\sim} \rightarrow b^{-}, a \oplus b^{\sim}=b \leadsto a, a^{-} \oplus b=a \rightarrow b$ and $a^{\sim} \oplus b=b^{-} \leadsto a^{\sim}$;

(iv) if $a, b \in M(A)$, then $a^{\sim} \oplus b^{\sim}=(b \odot a)^{\sim}$ and $a^{-} \oplus b^{-}=(b \odot a)^{-}$.

ProOF. (i) Apply Lemma 3.1 (ii).

(ii) Apply Lemma 3.1 (iv).

(iii) Apply (ii).

(iv) By (iii), (1) and (2), we have that $a^{\sim} \oplus b^{\sim}=b \rightsquigarrow a^{\sim}=b \rightsquigarrow(a \rightsquigarrow 0)=$ $(b \odot a) \rightsquigarrow 0=(b \odot a)^{\sim}$ and $a^{-} \oplus b^{-}=a \rightarrow b^{-}=a \rightarrow(b \rightarrow 0)=(b \odot a) \rightarrow$ $0=(b \odot a)^{-}$.

The following proposition extends a result from [19].

Proposition 3.3. Let $\mathbf{A}$ be a good pseudo-BL algebra. The structure $\mathbf{M}(\mathbf{A})=$ $\left(M(A), \oplus,^{-}, \sim, 0,1\right)$ is a pseudo-MV algebra. The order on $\mathrm{A}$ agrees with the one of $\mathbf{M}(\mathbf{A})$, defined by $a \leq_{M(A)} b$ if and only if $a^{-} \oplus b=1$.

PROOF. By Lemma 3.1 and Lemma 3.2, it follows that the operations $\oplus,^{-}, \sim$ are well defined on $M(A)$ and that $0,1 \in M(A)$. Let us denote by $\odot_{M(A)}$ the product on $M(A)$. Hence, for all $a, b \in M(A)$, we have that $a \odot_{M(A)} b=\left(b^{-} \oplus a^{-}\right)^{\sim}=$ 
$\left(b^{\sim} \oplus a^{\sim}\right)^{-} \in M(A)$. We shall verify the axioms from the definition of a pseudo-MV algebra. In the proof we use Lemma 3.1 and Lemma 3.2. Let $a, b, c \in M(A)$.

(i) We have that $(a \oplus b) \oplus c=\left(b^{\sim} . \odot a^{\sim}\right)^{-} \oplus\left(c^{\sim}\right)^{-}=\left(c^{\sim} \odot\left(b^{\sim} \odot a^{\sim}\right)\right)^{-}=$ $\left(\left(c^{\sim} \odot b^{\sim}\right) \odot a^{\sim}\right)^{-}=\left(a^{\sim}\right)^{-} \oplus\left(c^{\sim} \odot b^{\sim}\right)^{-}=a \oplus(b \oplus c)$. We also get that $a \oplus 0=\left(0^{\sim} \odot a^{\sim}\right)^{-}=\left(1 \odot a^{\sim}\right)^{-}=a^{\sim}=a$. Similarly, $0 \oplus a=a$. Hence $(M(A), \oplus, 0)$ is a monoid.

(ii) By (8), (11) and (12), $a \oplus 1=\left(1^{\sim} \odot a^{\sim}\right)^{-}=\left(0 \odot a^{\sim}\right)^{-}=0^{-}=1$. We obtain $1 \oplus a=1$ similarly.

(iii) Apply (12).

(iv) By $(*),\left(a^{-} \oplus b^{-}\right)^{\sim}=(b \odot a)^{\sim}=(b \odot a)^{\sim-}=\left(a^{\sim} \oplus b^{\sim}\right)^{-}$.

(v) We have to prove that $a \oplus\left(a^{\sim} \odot_{M(A)} b\right)=b \oplus\left(b^{\sim} \odot_{M(A)} a\right)=\left(a \odot_{M(A)} b^{-}\right) \oplus b=$ $\left(b \odot_{M(A)} a^{-}\right) \oplus a$. Applying (18) and (A4), we get that

$$
\begin{aligned}
a \oplus\left(a^{\sim} \odot_{M(A)} b\right) & =a \oplus\left(b^{-} \oplus a\right)^{\sim}=\left(a^{-}\right)^{\sim} \oplus\left(b^{-} \oplus a\right)^{\sim} \\
& =\left(\left(b^{-} \oplus a\right) \odot a^{-}\right)^{\sim}=\left((b \rightarrow a) \odot a^{-}\right)^{\sim} \\
& =\left(\left(a^{-} \leadsto b^{-}\right) \odot a^{-}\right)^{\sim}=\left(a^{-} \wedge b^{-}\right)^{\sim} \\
& =a^{-\sim} \vee b^{-\sim}=a \vee b
\end{aligned}
$$

and

$$
\begin{aligned}
\left(b \odot_{M(A)} a^{-}\right) \oplus a & =\left(a \oplus b^{\sim}\right)^{-} \oplus a=\left(a \oplus b^{\sim}\right)^{-} \oplus\left(a^{\sim}\right)^{-} \\
& =\left(a^{\sim} \odot\left(a \oplus b^{\sim}\right)\right)^{-}=\left(a^{\sim} \odot(b \leadsto a)\right)^{-} \\
& =\left(a^{\sim} \odot\left(a^{\sim} \rightarrow b^{\sim}\right)\right)^{-}=\left(a^{\sim} \wedge b^{\sim}\right)^{-} \\
& =a^{\sim-} \vee b^{\sim-}=a \vee b .
\end{aligned}
$$

Similarly we get $b \oplus\left(b^{\sim} \odot_{M(A)} a\right)=\left(a \odot_{M(A)} b^{-}\right) \oplus b=b \vee a=a \vee b$.

$$
\begin{aligned}
a \odot_{M(A)}\left(a^{-} \oplus b\right) & =a \odot_{M(A)}(a \rightarrow b)=\left((a \rightarrow b)^{-} \oplus a^{-}\right)^{\sim} \\
& =(a \odot(a \rightarrow b))^{-\sim}=(a \wedge b)^{-\sim}=(b \wedge a)^{-\sim} \\
& =((b \rightsquigarrow a) \odot b)^{-\sim}=\left(b^{-} \oplus(b \leadsto a)^{-}\right)^{\sim} \\
& =(b \rightsquigarrow a) \odot_{M(A)} b=\left(a \oplus b^{\sim}\right) \odot_{M(A)} b .
\end{aligned}
$$

(vii) It follows from the definition of $M(A)$.

Hence, M(A) is a pseudo-MV algebra. By Lemma 3.2 (iii), we have that for all $a, b \in M(A), a \leq_{M(A)} b$ if and only if $b \oplus a^{\sim}=1$ if and only if $a \rightsquigarrow b=1$ if and only if $a \leq b$.

As a consequence of this proposition we obtain [4, Corollary 2.34]:

COROLlaRY 3.4. A pseudo-BL algebra $\mathrm{A}$ is a pseudo-MV algebra if and only if $a^{\sim-}=a^{-\sim}=$ a for all $a \in A$. 
REMARK 3.5. Let $\mathbf{A}$ be a good pseudo-BL algebra. For any $a, b \in M(A)$,

$$
a \odot_{M(A)} b=\left(b^{\sim} \oplus a^{\sim}\right)^{-}=\left(b^{-} \oplus a^{-}\right)^{\sim}=(a \odot b)^{\sim-}=(a \odot b)^{-\sim} .
$$

PROOF. Apply the definitions of $\oplus$ and $\odot_{M(A)}$.

Since, $a, b \in M(A)$ does not imply $a \odot b \in M(A)$, it follows that, generally, $(a \odot b)^{-\sim} \neq a \odot b$. Hence, the product on the pseudo-MV algebra $\mathbf{M}(\mathbf{A})$ does not coincide with the product on the pseudo-BL algebra $\mathrm{A}$. In the case of BL-algebras, the product is the same (see [19]).

PROPOSITION 3.6. Let $\mathrm{A}$ be a good pseudo-BL algebra. Then $\mathrm{A}$ is a strong pseudo$\mathrm{BL}$ algebra if and only if $\mathrm{M}(\mathrm{A})$ is a strong pseudo-MV algebra.

PROOF. If $a^{\sim}=a^{-}$for all $a \in A$, then $a^{\sim}=a^{-}$for all $a \in M(A)$. Conversely, suppose that $a^{\sim}=a^{-}$for all $a \in M(A)$. Let $a \in A$. By (16) and (*), $a^{\sim}=$ $a^{\sim \sim}=\left(a^{\sim-}\right)^{\sim}=\left(a^{-\sim}\right)^{\sim}$. But, by Lemma 3.1 (ii), we have that $a^{-\sim} \in M(A)$, so $\left(a^{-\sim}\right)^{\sim}=\left(a^{-\sim}\right)^{-}=a^{-}$, by (16). Thus, for all $a \in A$, we have that $a^{\sim}=a^{-}$.

Let $\mathbf{A}$ be a good pseudo-BL algebra. Since, by Lemma 3.1 (ii), $a^{-}, a^{\sim} \in M(A)$ for any $a \in A$, we can define the maps $\varphi_{1}: A \rightarrow M(A)$ by $\varphi_{1}(a)=a^{\sim}$ for any $a \in A$, and $\varphi_{2}: A \rightarrow M(A)$ by $\varphi_{2}(a)=a^{-}$for any $a \in A$.

LEMMA 3.7. Let A be a good pseudo-BL algebra. The following properties hold for all $a, b \in A$ :

(i) $\varphi_{1}, \varphi_{2}$ are onto;

(ii) $\varphi_{1}(a \vee b)=\varphi_{1}(a) \wedge \varphi_{1}(b)$ and $\varphi_{2}(a \vee b)=\varphi_{2}(a) \wedge \varphi_{2}(b)$;

(iii) $\varphi_{1}(a \wedge b)=\varphi_{1}(a) \vee \varphi_{1}(b)$ and $\varphi_{2}(a \wedge b)=\varphi_{2}(a) \vee \varphi_{2}(b)$;

(iv) $\varphi_{1}(a) \leq \varphi_{1}(b)$ if and only if $\varphi_{2}(a) \leq \varphi_{2}(b)$;

(v) $a \leq b$ implies $\varphi_{1}(a) \geq \varphi_{1}(b)$ and $\varphi_{2}(a) \geq \varphi_{2}(b)$;

(vi) $\varphi_{1}(a)=1$ if and only if $\varphi_{2}(a)=1$ if and only if $a=0$;

(vii) $\varphi_{1}(1)=\varphi_{2}(1)=0$;

(viii) $\varphi_{1}(a)=0$ if and only if $\varphi_{2}(a)=0$;

(ix) $\varphi_{1}(a \odot b)=\varphi_{1}(b) \oplus \varphi_{1}(a)$ and $\varphi_{2}(a \odot b)=\varphi_{2}(b) \oplus \varphi_{2}(a)$;

(x) for any $n \in \omega, \varphi_{1}\left(a^{n}\right)=n \varphi_{1}(a)$ and $\varphi_{2}\left(a^{n}\right)=n \varphi_{2}(a)$.

PROOF. (i) Let $a \in M(A)$. Then $a=a^{-\sim}=\varphi_{1}\left(a^{-}\right)$and $a=a^{\sim-}=\varphi_{2}\left(a^{\sim}\right)$.

(ii) Apply (18).

(iii) Apply (19).

(iv) Suppose that $\varphi_{1}(a) \leq \varphi_{1}(b)$, that is, $a^{\sim} \leq b^{\sim}$. Applying (13) and (16), it follows that $b^{-\sim}=b^{\sim-} \leq a^{\sim-}=a^{-\sim}$, so $a^{-}=a^{-\sim-} \leq b^{-\sim-}=b^{-}$. Hence, $\varphi_{2}(a) \leq \varphi_{2}(b)$. We prove similarly that $\varphi_{2}(a) \leq \varphi_{2}(b)$ implies $\varphi_{1}(a) \leq \varphi_{1}(b)$. 
(v) Apply (13).

(vi) Apply (11).

(vii) Apply (12).

(viii) Suppose that $a^{\sim}=0$, so $a^{-\sim}=a^{\sim-}=1$, hence, $a^{-}=a^{-\sim-}=0$. We get similarly that $a^{-}=0$ implies $a^{\sim}=0$.

(ix) Apply Lemma 3.2 (iv).

(x) By induction on $n$. For $n=0$, we have that $a^{0}=1$, so $\varphi_{1}(1)=0$ and $0 \varphi_{1}(a)=0$. Suppose that $\varphi_{1}\left(a^{n}\right)=n \varphi_{1}(a)$. By (ix), it follows that $\varphi_{1}\left(a^{n+1}\right)=$ $\varphi_{1}\left(a^{n} \odot a\right)=\varphi_{1}(a) \oplus \varphi_{1}\left(a^{n}\right)=\varphi_{1}(a) \oplus n \varphi_{1}(a)=(n+1) \varphi_{1}(a)$. Similarly for $\varphi_{2}$.

LEMMA 3.8. Let A be a nontrivial good pseudo-BL algebra. Then

(i) $A_{0}^{-}=A_{0}^{\sim} \stackrel{\text { not }}{=} A_{0}$;

(ii) if $a \in A_{0}$, then $\operatorname{ord}(a)=\infty$;

(iii) for any $a \in A$, ord $(a)=\mathrm{MV}$-ord $\left(\varphi_{1}(a)\right)=\mathrm{MV}-\operatorname{ord}\left(\varphi_{2}(a)\right)$;

(iv) for any $a \in A$, ord $\left(a^{\sim}\right)=\operatorname{ord}\left(a^{-}\right)$;

(v) $\varphi_{1}(D(A))=\varphi_{2}(D(A))=D(M(A))$ and $\varphi_{1}^{-1}(D(M(A)))=\varphi_{2}^{-1}(D(M(A)))$ $=D(A)$.

ProOF. (i) Apply Lemma 3.7 (viii).

(ii) Suppose that there is $n \in \omega$ such that $a^{n}=0$. Then, applying Lemma 3.7 (vi) and $(\mathrm{x})$, we get that $\varphi_{1}\left(a^{n}\right)=\varphi_{1}(0)=1$ and $\varphi_{1}\left(a^{n}\right)=n \varphi_{1}(a)=n a^{\sim}=n 0=0$. We get that $0=1$, a contradiction. Hence, $a^{n} \neq 0$ for all $n \in \omega$, so ord $(a)=\infty$.

(iii) Let $a \in A$ and $n \in \omega$. By Lemma 3.7 (vi), we have that $a^{n}=0$ if and only if $\varphi_{1}\left(a^{n}\right)=1$ if and only if $n \varphi_{1}(a)=1$. Hence, $\operatorname{ord}(a)=\mathrm{MV}$-ord $\left(\varphi_{1}(a)\right)$. Similarly for $\varphi_{2}$.

(iv) Let $a \in A$. Applying (i) and (*), we get that $\operatorname{ord}\left(a^{\sim}\right)=\operatorname{MV-ord}\left(\varphi_{2}\left(a^{\sim}\right)\right)=$ $\mathrm{MV}$-ord $\left(a^{--}\right)=\mathrm{MV}$-ord $\left(a^{-\sim}\right)=\mathrm{MV}$-ord $\left(\varphi_{1}\left(a^{-}\right)\right)=\operatorname{ord}\left(a^{-}\right)$.

(v) Apply (iii) and the fact that $\varphi_{1}, \varphi_{2}$ are onto.

LEMMA 3.9. Let $\mathbf{A}$ be a good pseudo-BL algebra. Suppose that I is an ideal of $\mathbf{M}(\mathbf{A})$ and $F$ is a filter of $\mathbf{A}$. Then

(i) $\varphi_{1}^{-1}(I), \varphi_{2}^{-1}(I)$ are filters of $\mathbf{A}$;

(ii) $\varphi_{1}(F), \varphi_{2}(F)$ are ideals of $\mathbf{M}(\mathbf{A})$;

(iii) $F \subseteq \varphi_{1}^{-1}\left(\varphi_{1}(F)\right)$ and $F \subseteq \varphi_{2}^{-1}\left(\varphi_{2}(F)\right)$;

(iv) $l=\varphi_{1}\left(\varphi_{1}^{-1}(I)\right)$ and $I=\varphi_{2}\left(\varphi_{2}^{-1}(I)\right)$;

(v) $I$ is proper if and only if $\varphi_{1}^{-1}(I)$ is proper if and only if $\varphi_{2}^{-1}(I)$ is proper;

(vi) $F$ is proper if and only if $\varphi_{1}(F)$ is proper if and only if $\varphi_{2}(F)$ is proper;

(vii) if $F$ is an ultrafilter of $\mathbf{A}$, then $F=\varphi_{1}^{-1}\left(\varphi_{1}(F)\right)$ and $F=\varphi_{2}^{-1}\left(\varphi_{2}(F)\right)$;

(viii) if $I$ is a maximal ideal of $\mathbf{M}(\mathbf{A})$, then $\varphi_{1}^{-1}(I), \varphi_{2}^{-1}(I)$ are ultrafilters of $\mathbf{A}$;

(ix) if $F$ is an ultrafilter of $\mathbf{A}$, then $\varphi_{1}(F), \varphi_{2}(F)$ are maximal ideals of $\mathbf{M}(\mathbf{A})$. 
PROOF. (i) Let us prove that $\varphi_{1}^{-1}(I)$ is a filter of A. Since $\varphi_{1}(1)=0 \in I$, we have $1 \in \varphi_{1}^{-1}(I)$. Let $a_{1}, a_{2} \in \varphi_{1}^{-1}(I)$. It follows that $\varphi_{1}\left(a_{1}\right), \varphi_{1}\left(a_{2}\right) \in I$, so $\varphi_{1}\left(a_{1} \odot a_{2}\right)=\varphi_{1}\left(a_{2}\right) \oplus \varphi_{1}\left(a_{1}\right) \in I$. Hence, $a_{1} \odot a_{2} \in \varphi_{1}^{-1}(I)$. Let $a_{1} \in \varphi_{1}^{-1}(I)$, $a_{2} \in A$ be such that $a_{1} \leq a_{2}$. By Lemma $3.7(\mathrm{v})$, we get $\varphi_{1}\left(a_{2}\right) \leq \varphi_{1}\left(a_{1}\right) \in I$, so $\varphi_{1}\left(a_{2}\right) \in I$, that is, $a_{2} \in \varphi_{1}^{-1}(I)$. Thus, we have proved that $\varphi_{1}^{-1}(I)$ is a filter of $\mathbf{A}$. We get similarly that $\varphi_{2}^{-1}(I)$ is a filter of $\mathbf{A}$.

(ii) Let us prove that $\varphi_{1}(F)$ is an ideal of $\mathbf{M}(\mathbf{A})$. We have that $0=\varphi_{1}(1) \in \varphi_{1}(F)$. Let $b_{1}, b_{2} \in \varphi_{1}(F)$. That is, there are $a_{1}, a_{2} \in F$ such that $b_{1}=\varphi_{1}\left(a_{1}\right)$ and $b_{2}=\varphi_{1}\left(a_{2}\right)$. We have $a_{2} \odot a_{1} \in F$ and $b_{1} \oplus b_{2}=\varphi_{1}\left(a_{2} \odot a_{1}\right) \in \varphi_{1}(F)$. Let $b_{1}, b_{2} \in M(A)$ be such that $b_{1} \leq b_{2}$ and $b_{2} \in \varphi_{1}(F)$. It follows that $b_{2}=\varphi_{1}\left(a_{2}\right)$ with $a_{2} \in F$ and, since $\varphi_{1}$ is onto, there is $a \in A$ such that $\varphi_{1}(a)=b_{1}$. Let $a_{1}=a \vee a_{2}$. Then $a_{2} \leq a_{1}$ and $a_{2} \in F$, so $a_{1} \in F$ and $\varphi_{1}\left(a_{1}\right)=\varphi_{1}(a) \wedge \varphi_{1}\left(a_{2}\right)=b_{1} \wedge b_{2}=b_{1}$, by Lemma 3.7 (iii). Hence, $b_{1} \in \varphi_{1}(F)$. We obtain in the same manner that $\varphi_{2}(F)$ is an ideal of $\mathbf{M}(\mathbf{A})$.

(iii) It is obvious.

(iv) It follows from the fact that $\varphi_{1}$ and $\varphi_{2}$ are onto.

(v) $I$ is not proper if and only if $1 \in I$ if and only if $\varphi_{1}(0) \in I$ if and only if $0 \in \varphi_{1}^{-1}(I)$ if and only if $\varphi_{1}^{-1}(I)$ is not proper.

(vi) If $0 \in F$, then $1=\varphi_{1}(0) \in \varphi_{1}(F)$. Suppose that $1 \in \varphi_{1}(F)$. Then, there is $a \in F$ such that $\varphi_{1}(a)=1$. Applying Lemma 3.7 (vi), we get that $a=0$, hence $0 \in F$.

(vii) Suppose that $F$ is an ultrafilter of $\mathbf{A}$. Then, by (v) and (vi), $\varphi_{1}^{-1}\left(\varphi_{1}(F)\right)$ is a proper filter of $\mathbf{A}$ and, by (iii), $F \subseteq \varphi_{1}^{-1}\left(\varphi_{1}(F)\right)$. Since $F$ is ultrafilter, we get that $F=\varphi_{1}^{-1}\left(\varphi_{1}(F)\right)$.

(viii) Suppose that $\varphi_{1}^{-1}(I) \subseteq F$, where $F$ is a proper filter of $\mathbf{A}$. It follows that $I=\varphi_{1}\left(\varphi_{1}^{-1}(I)\right) \subseteq \varphi_{1}(F)$. Since $\varphi_{1}(F)$ is proper, we get that $I=\varphi_{1}(F)$, so $\varphi_{1}^{-1}(I)=\varphi_{1}^{-1}\left(\varphi_{1}(F)\right) \supseteq F$. Hence, $\varphi_{1}^{-1}(I)=F$.

(ix) Suppose that $\varphi_{1}(F) \subseteq I$, where $I$ is a proper ideal of $\mathbf{M}(\mathbf{A})$. It follows that $F=\varphi_{1}^{-1}(\varphi(F)) \subseteq \varphi_{1}^{-1}(I)$. Since $\varphi_{1}^{-1}(I)$ is proper, we get that $F=\varphi_{1}^{-1}(I)$, so $\varphi_{1}(F)=\varphi_{1}\left(\varphi_{1}^{-1}(I)\right)=I$.

The next result is a consequence of the above proposition.

PROPOSITION 3.10. The maps $\varphi_{1}, \varphi_{2}$ are bijections between the set of ultrafilters of $\mathbf{A}$ and the set of maximal ideals of $\mathbf{M}(\mathbf{A})$.

COROLLARY 3.11. Let $\mathbf{A}$ be a good pseudo-BL algebra. Then $\mathbf{A}$ is local if and only if $\mathbf{M}(\mathbf{A})$ is local.

We remark that if $\mathbf{A}$ is a BL-algebra, then $\varphi_{1}=\varphi_{2}$ and the results obtained above extend some results from $[19,8]$. 
PROPOSITION 3.12. Let A be a good pseudo-BL algebra. Suppose that $I$ is an ideal of $\mathbf{M}(\mathbf{A})$ and $F$ is a filter of $\mathbf{A}$. Then

(i) if $F$ is normal in $\mathbf{A}$, then $\varphi_{1}(F)=\varphi_{2}(F) \stackrel{\text { not }}{=} \varphi(F)$;

(ii) if $F$ is normal in $\mathbf{A}$, then $\varphi(F)$ is normal in $\mathbf{M}(\mathbf{A})$;

(iii) if $I$ is normal in $\mathbf{M}(\mathbf{A})$, then $\varphi_{1}^{-1}(I)=\varphi_{2}^{-1}(I)$.

PROOF. (i) Let $b \in \varphi_{1}(F)$, that is, $b=\varphi_{1}(a)$ with $a \in F$. By (14), we have that $a \leq a^{\sim-}$, so $a^{\sim-} \in F$, hence $a^{\approx} \in F$, since $F$ is a normal filter of $\mathbf{A}$. Since $b \in M(A)$, we also get that $b=b^{\sim-}=a^{\approx-}=\varphi_{2}\left(a^{\approx}\right)$, hence $b \in \varphi_{2}(F)$. Thus, $\varphi_{1}(F) \subseteq \varphi_{2}(F)$. The other inclusion is proved similarly.

(ii) Let $b, c \in M(A)$. By Lemma 3.2 (iii), we have $b^{\sim} \odot_{M(A)} c=\left(c^{-} \oplus b\right)^{\sim}=$ $(c \rightarrow b)^{\sim}$ and $c \odot_{M(A)} b^{-}=\left(b \oplus c^{\sim}\right)^{-}=(c \leadsto b)^{-}$. Suppose that $b^{\sim} \odot_{M(A)} c \in \varphi(F)$, so there is $a \in F$ such that $(c \rightarrow b)^{\sim}=a^{\sim}$. But $c \rightarrow b=c^{-} \oplus b \in M(A)$, hence $c \rightarrow b=(c \rightarrow b)^{\sim-}=a^{\sim-} \geq a$, by (14). Since $a \in F$ and $F$ is a filter, we get that $c \rightarrow b \in F$. But $F$ is normal, hence $c \rightsquigarrow b \in F$. We obtain that $c \odot_{M(A)} b^{-}=(c \rightsquigarrow b)^{-} \in \varphi(F)$. We get similarly that $c \odot_{M(A)} b^{-} \in \varphi(F)$ implies $b^{\sim} \odot_{M(A)} c \in \varphi(F)$.

(iii) Let $a \in \varphi_{1}^{-1}(I)$, so $a^{\sim} \in I$. Since $I$ is normal, from $a^{\sim} \in I$ and Lemma 1.23 we get that $a^{\sim=} \in I$. But, by $(*)$ and (16), $a^{\sim}=a^{-\sim-}=a^{-}=\varphi_{2}(a)$. We have got that $\varphi_{2}(a) \in I$, that is $a \in \varphi_{2}^{-1}(I)$. We prove similarly that $a \in \varphi_{2}^{-1}(I)$ implies $a \in \varphi_{1}^{-1}(I)$.

\section{Some classes of local pseudo-BL algebras}

\section{Perfect pseudo-BL algebras A pseudo-BL algebra $\mathbf{A}$ is called perfect if}

(i) $\mathbf{A}$ is a local good pseudo-BL algebra, and

(ii) for any $a \in A$, ord $(a)<\infty$ if and only if $\operatorname{ord}\left(a^{\sim}\right)=\infty$.

Proposition 4.1. Let $\mathbf{A}$ be a good pseudo-BL algebra. Then $\mathbf{A}$ is perfect if and only if $\mathbf{M}(\mathbf{A})$ is perfect.

PROOF. We have that $\mathbf{A}$ is local if and only if $\mathbf{M}(\mathbf{A})$ is local, by Corollary 3.11 . In the sequel, we shall apply repeatedly Proposition 3.8 (iii). Suppose that $\mathbf{A}$ is perfect and let $a \in M(A)$, so $a=a^{\sim-}$. We get that MV-ord $(a)<\infty$ if and only if MV-ord $\left(a^{\sim}\right)<\infty$ if and only if $\operatorname{ord}\left(a^{\sim}\right)<\infty$ if and only if ord $(a)=\infty$ if and only if $\mathrm{MV}-\operatorname{ord}\left(a^{\sim}\right)=\infty$. Hence, $\mathbf{M}(\mathbf{A})$ is perfect. Conversely, suppose that $\mathbf{M}(\mathbf{A})$ is perfect and let $a \in A$. Then, by Lemma 3.1 (i), $a^{\sim} \in M(A)$. It follows that $\operatorname{ord}(a)<\infty$ if and only if MV-ord $\left(a^{\sim}\right)<\infty$ if and only if MV-ord $\left(a^{\approx}\right)=\infty$ if and only if $\operatorname{ord}\left(a^{\sim}\right)=\infty$. Hence, $\mathbf{A}$ is perfect. 
PROPOSITION 4.2. Let $\mathbf{A}$ be a local good pseudo-BL algebra. The following are equivalent:

(i) A is perfect;

(ii) for any $a \in A$, ord $(a)<\infty$ implies $\operatorname{ord}\left(a^{\sim}\right)=\infty$;

(ii') for any $a \in A$, ord $(a)<\infty$ implies $\operatorname{ord}\left(a^{-}\right)=\infty$;

(iii) $D(A)_{\sim}^{*}=D(A)^{*}$;

(iii') $D(A)_{-}^{*}=D(A)^{*}$.

PROOF. (i) if and only if (ii). Let $a \in A$. Since $\mathbf{A}$ is local, by Corollary 2.3, we have that $\operatorname{ord}(a)=\infty$ implies ord $\left(a^{\sim}\right)<\infty$, hence $\operatorname{ord}\left(a^{\sim}\right)=\infty$ implies ord $(a)<\infty$. It follows that $\mathbf{A}$ is perfect if and only if (ord $(a)<\infty$ implies $\operatorname{ord}\left(a^{\sim}\right)=\infty$ ).

(ii) if and only if (ii'). Apply Lemma 3.8 (iv).

(ii') implies (iii). Since $\mathbf{A}$ is local, $D(A)_{\sim}^{*} \subseteq D(A)^{*}$, by Corollary 2.3 (ii). Let us prove the converse inclusion. Let $a \in A$ be such that ord $(a)<\infty$. From (ii') we get that ord $\left(a^{-}\right)=\infty$, so $a^{-} \in D(A)$ and, by (14), $a \leq a^{-\sim}$. Hence, $a \in D(A)_{\sim}^{*}$.

(iii) implies (ii'). Suppose that $D(A)_{\sim}^{*}=D(A)^{*}$ and let $a \in A$ with $\operatorname{ord}(a)<\infty$, that is, $a \in D(A)^{*}$. It follows that there is $x \in D(A)$ such that $a \leq x^{\sim}$, so $x^{\sim-} \leq a^{-}$, by (13). Since $x \leq x^{\sim-}$ and $\operatorname{ord}(x)=\infty$, applying Lemma 1.7 (iii), we get that $\operatorname{ord}\left(x^{\sim-}\right)=\infty$. Applying again Lemma 1.7 (iii), from $x^{\sim-} \leq a^{-}$it follows that $\operatorname{ord}\left(a^{-}\right)=\infty$.

(ii) if and only if (iii'). It is similar to '(ii') if and only (iii)'.

A primary filter $P$ of a pseudo-BL algebra $\mathbf{A}$ is called perfect if for all $a \in A$, $\left(a^{n}\right)^{\sim} \in P$ for some $n \in \omega$ implies $\left(\left(a^{\sim}\right)^{m}\right)^{\sim} \notin P$ for all $m \in \omega$.

LEMMA 4.3. Let $\mathbf{A}$ be a pseudo-BL algebra and $P$ be a perfect filter of $\mathbf{A}$. Then for all $a \in A,\left(a^{n}\right)^{\sim} \in P$ for some $n \in \omega$ if and only if $\left(\left(a^{\sim}\right)^{m}\right)^{\sim} \notin P$ for all $m \in \omega$.

PROOF. Let $a \in A$ such that $\left(\left(a^{\sim}\right)^{m}\right)^{\sim} \notin P$ for all $m \in \omega$. We have to prove that $\left(a^{n}\right)^{\sim} \in P$ for some $n \in \omega$. By $(9), a^{\sim} \odot a=0$, hence $\left(\left(a^{\sim} \odot a\right)^{n}\right)^{\sim}=0^{\sim}=1 \in P$ for all $n \in \omega$. Apply now the fact that $P$ is primary and the hypothesis to get that $\left(a^{n}\right)^{\sim} \in P$ for some $n \in \omega$.

PROPOSITION 4.4. Let $\mathbf{A}$ be a good pseudo-BL algebra and $P$ be a proper normal filter of $\mathbf{A}$. The following are equivalent:

(i) $\mathbf{A} / P$ is a perfect pseudo-BL algebra;

(ii) $P$ is a perfect filter of $\mathbf{A}$;

(iii) $P$ is primary and for all $a \in A,\left(a^{n}\right)^{-} \in P$ for some $n \in \omega$ implies $\left(\left(a^{-}\right)^{m}\right)^{-} \notin$ $P$ for all $m \in \omega$. 
PROOF. Since good pseudo-BL algebras form a variety, it follows that $\mathbf{A} / \boldsymbol{P}$ is a good pseudo-BL algebra. By Proposition 2.6, we have that $\mathbf{A} / P$ is local if and only if $P$ is primary. Let $a \in A$. Applying Lemma 1.10, we get that $\operatorname{ord}(a / P)<\infty$ if and only if $(a / P)^{n}=0 / P$ for some $n \in \omega$ if and only if $\left(a^{n}\right)^{\sim} \in P$ for some $n \in \omega$ if and only if $\left(a^{n}\right)^{-} \in P$ for some $n \in \omega$, that $\operatorname{ord}\left((a / P)^{\sim}\right)=\infty$ if and only if $\left((a / P)^{\sim}\right)^{m} \neq 0 / P$ for all $m \in \omega$ if and only if $\left(\left(a^{\sim}\right)^{m}\right)^{\sim} \notin P$ for all $m \in \omega$ and that $\operatorname{ord}\left((a / P)^{-}\right)=\infty$ if and only if $\left((a / P)^{-}\right)^{m} \neq 0 / P$ for all $m \in \omega$ if and only if $\left(\left(a^{-}\right)^{m}\right)^{-} \notin P$ for all $m \in \omega$. Apply now Proposition 4.2 (ii) and (iii) to get that (i) if and only if (ii) and (i) if and only if (iii).

PROPOSITION 4.5. Let $\mathrm{A}$ be $a \mathrm{BL}$-algebra and $P$ be a proper filter of $\mathbf{A}$. The following are equivalent:

(i) $P$ is a perfect filter of $\mathbf{A}$;

(ii) for all $a \in A,\left(a^{n}\right)^{-} \in P$ for some $n \in \omega$ if and only if $\left(\left(a^{-}\right)^{m}\right)^{-} \notin P$ for all $m \in \omega$.

ProOF. (i) implies (ii). Apply Lemma 4.3.

(ii) implies (i). We shall prove that $A / P$ is local and apply Proposition 2.6 to get that $P$ is a primary filter. Let $a \in A$ and suppose that $\operatorname{ord}\left(a^{-} / P\right)=\infty$. As in the proof of Proposition 4.4, we get $\left(\left(a^{-}\right)^{m}\right)^{-} \notin P$ for all $m \in \omega$. Applying (i), it follows that $\left(a^{n}\right)^{-} \in P$ for some $n \in \omega$, that is, ord $(a / P)<\infty$. Thus, we have proved that for all $a \in A, \operatorname{ord}(a / P)<\infty$ or ord $\left(a^{-} / P\right)<\infty$. Apply now [19, Proposition 1] to obtain that $\mathbf{A} / P$ is local.

Hence, in the case that $\mathbf{A}$ is a BL-algebra, the notion of perfect filter defined above coincides with the notion of perfect filter defined in [19].

PROPOSITION 4.6. Let $\mathrm{A}$ be a good pseudo-BL algebra. The following are equivalent:

(i) A is perfect;

(ii) any proper normal filter of $\mathbf{A}$ is perfect;

(iii) $\{1\}$ is a perfect filter of $\mathbf{A}$.

Proof. (i) implies (ii). Let $F$ be a proper normal filter of $\mathbf{A}$. Since $\mathbf{A}$ is local, by Proposition 2.9 it follows that $F$ is primary. Let $a \in A$ such that $\left(a^{n}\right)^{\sim} \in F$ for some $n \in \omega$. Suppose that $\left(\left(a^{\sim}\right)^{k}\right)^{\sim} \in F$ for some $k \in \omega$. We get that $\left\langle\left(a^{n}\right)^{\sim}\right\rangle,\left\langle\left(\left(a^{\sim}\right)^{k}\right)^{\sim}\right\rangle \subseteq$ $F$ and, since $F$ is proper, it follows that $\left\langle\left(a^{n}\right)^{\sim}\right\rangle$ and $\left\langle\left(\left(a^{\sim}\right)^{k}\right)^{\sim}\right\rangle$ are also proper filters of A. Applying Lemma 1.7 (i), we get that ord $\left(\left(a^{n}\right)^{\sim}\right)=\operatorname{ord}\left(\left(\left(a^{\sim}\right)^{k}\right)^{\sim}\right)=\infty$. Since A is perfect, we obtain that ord $\left(a^{n}\right)<\infty$ and $\operatorname{ord}\left(\left(a^{\sim}\right)^{k}\right)<\infty$, hence, $\operatorname{ord}(a)<\infty$ and $\operatorname{ord}\left(a^{\sim}\right)<\infty$, a contradiction with the fact that $\mathbf{A}$ is perfect. Thus, $\left(a^{n}\right)^{\sim} \in F$ for some $n \in \omega$ implies $\left(\left(a^{\sim}\right)^{m}\right)^{\sim} \notin F$ for all $m \in \omega$. 
(ii) implies (iii). It is obvious, since $\{1\}$ is a proper normal filter of $\mathbf{A}$.

(iii) implies (i). Since $\{1\}$ is a perfect filter of $\mathbf{A}$, applying Proposition 4.4, we get that $\mathbf{A} /\{1\}$ is perfect. But $\mathbf{A} \cong \mathbf{A} /\{1\}$, hence $\mathbf{A}$ is perfect.

Locally finite pseudo-BL algebras According to [5], a pseudo-BL algebra $\mathbf{A}$ is locally finite if for any $a \in A, a \neq 1$ implies ord $(a)<\infty$.

PROPOSITION 4.7. Let $\mathrm{A}$ be a pseudo-BL algebra. The following are equivalent:

(i) A is locally finite;

(ii) $\{1\}$ is the unique proper filter of $\mathbf{A}$.

PROOF. Applying Lemma 1.7 (i), it follows that $A$ is locally finite if and only if for every $a \in A$, if $a \neq 1$ then $\langle a\rangle=A$ if and only if $\{1\}$ is the unique proper filter of $A$.

PROPOSITION 4.8. Every locally finite pseudo-BL algebra $\mathrm{A}$ is a local pseudo-BL algebra.

Proof. We have that $D(A)=\{1\}$, hence $D(A)$ is a filter of A. Apply Proposition 2.2 to get that $\mathbf{A}$ is local.

In [5] it is proved that locally finite pseudo-BL algebras are locally finite MValgebras. We shall give a simpler proof of this fact.

PROPOSITION 4.9. Let $\mathbf{A}$ be a locally finite pseudo-BL algebra. Then for all $a \in A$, $a^{\sim-}=a^{-\sim}=$ a. Hence, $A=M(A)$.

PROOF. If $a=0$, then it follows immediately that $0^{\sim-}=0^{-\sim}=0$. Suppose that $a \neq 0$. Let us prove that $a^{-\sim}=a$. By (14), we have that $a \leq a^{-\sim}$. Suppose that $a^{-\sim} \notin a$, hence $a^{-\sim} \rightarrow a \neq 1$. Since $\mathbf{A}$ is locally finite, it follows that $\operatorname{ord}\left(a^{-\sim} \rightarrow a\right)<\infty$, hence $\left(a^{-\sim} \rightarrow a\right)^{n}=0$ for some $n \in \omega-\{0\}$. By (16), (2), (A4) and (14), we get

$$
\begin{aligned}
\left(a^{-\sim} \rightarrow a\right) \rightarrow a^{-} & =\left(a^{-\sim} \rightarrow a\right) \rightarrow a^{-\sim}=\left(a^{-\sim} \rightarrow a\right) \rightarrow\left(a^{-\sim} \rightarrow 0\right) \\
& =a^{-\sim} \odot\left(a^{-\sim} \rightarrow a\right) \rightarrow 0=\left(a \wedge a^{-\sim}\right) \rightarrow 0=a \rightarrow 0=a^{-} .
\end{aligned}
$$

Applying repeatedly this procedure, it follows that $\left(a^{-\sim} \rightarrow a\right)^{n} \rightarrow a^{-}=a^{-}$, hence $a^{-}=0 \rightarrow a^{-}=1$, so, by (11), $a=0$. We have got a contradiction, since $a \neq 0$. Hence, $a^{-\sim}=a$. We prove similarly that $a^{\sim-}=a$. 
COROLLARY 4.10 ([5]). Every locally finite pseudo-BL algebra A is a locally finite MV-algebra.

Proof. Applying Proposition 4.9 and Proposition 1.24, we get that $\mathbf{A}$ is a pseudoMV algebra. Let $a \in A, a \neq 0$, so $a^{\sim} \neq 1$, by (11). By Proposition 3.8 (i), we obtain that MV-ord $(a)=\mathrm{MV}-\operatorname{ord}\left(a^{\sim-}\right)=\operatorname{ord}\left(a^{\sim}\right)<\infty$. Thus, we have proved that $\mathbf{A}$ is a locally finite pseudo-MV algebra. Apply now [15, Proposition 39] to get that $\mathbf{A}$ is a locally-finite MV-algebra.

Peculiar pseudo-BL algebras A pseudo-BL algebra $\mathbf{A}$ is called peculiar if

(i) $\mathbf{A}$ is a local good pseudo-BL algebra;

(ii) there is $a \in A-\{1\}$ such that $\operatorname{ord}(a)=\infty$;

(iii) there is $a \in A$ such that $\operatorname{ord}(a)<\infty$ and $\operatorname{ord}\left(a^{\sim}\right)<\infty$.

Let us denote by $\mathscr{P} \mathscr{F}$ the class of perfect pseudo-BL algebras, by $\mathscr{L} \mathscr{F}$ the class of locally finite pseudo-BL algebras and by $\mathscr{P} \mathscr{C}$ the class of peculiar pseudo-BL algebras. The following proposition is similar to [2, Theorem 5.1].

PROPOSITION 4.11. Let $\mathbf{A}$ be a local good pseudo-BL algebra different from $\mathbf{L}_{2}=$ $\{0,1\}$. Then exactly one of the following holds:

(i) $\mathbf{A} \in \mathscr{P} \mathscr{F}$;

(ii) $\mathbf{A} \in \mathscr{L} \mathscr{F}$;

(iii) $\mathbf{A} \in \mathscr{P} \mathscr{C}$.

PROOF. By the definitions, if $\mathbf{A} \notin \mathscr{P} \mathscr{F} \cup \mathscr{L} \mathscr{F}$, then $\mathbf{A} \in \mathscr{P} \mathscr{C}$. Hence, one of (i), (ii) or (iii) holds. It is easy to see that $\mathscr{P} \mathscr{C} \cap \mathscr{L} \mathscr{F}=\mathscr{P} \mathscr{C} \cap \mathscr{P} \mathscr{F}=\emptyset$. Let us prove that $\mathscr{P} \mathscr{F} \cap \mathscr{L} \mathscr{F}=\left\{\mathbf{L}_{2}\right\}$. Obviously, $\mathbf{L}_{2}$ is perfect and locally finite. Now, let $A \neq \mathbf{L}_{2}$ be a locally finite pseudo-BL algebra. Since $A \neq\{0,1\}$, there is $a \in A$ such that $a \neq 0$ and $a \neq 1$. From $a \neq 0$ and (11) we get that $a^{\sim} \neq 1$. Applying now the fact that $\mathbf{A}$ is locally finite, it follows that $\operatorname{ord}(a)<\infty$ and $\operatorname{ord}\left(a^{\sim}\right)<\infty$. Hence, $\mathbf{A}$ is not perfect. That is, exactly one of (i), (ii), (iii) holds.

PROPOSITION 4.12. Let $\mathbf{A}$ be a locally good pseudo-BL algebra such that $A \neq$ $M(A)$. Then $\mathbf{A}$ is a peculiar pseudo-BL algebra if and only if $\mathbf{M}(\mathbf{A}) \neq \mathbf{L}_{2}$ is a singular pseudo-MV algebra.

Proof. Suppose that $\mathbf{A}$ is peculiar. Then $\mathbf{A}$ is not perfect, hence, by Proposition 4.1, $M(A)$ is not a perfect pseudo-MV algebra. Since $L_{2}$ is a perfect pseudo-MV algebra, it follows that $\mathbf{M}(\mathbf{A}) \neq \mathbf{L}_{2}$. Applying Proposition 1.25, we also get that $\mathbf{M}(\mathbf{A})$ is singular. Conversely, suppose that $\mathbf{M}(\mathbf{A}) \neq \mathbf{L}_{2}$ and that $\mathbf{M ( A )}$ is a singular pseudoMV algebra. Since $A \neq M(A)$, by Proposition 4.9 we get that $\mathbf{A}$ is not locally 
finite. We also have that $\mathbf{M}(\mathbf{A})$ is not perfect, hence $\mathbf{A}$ is not perfect. Applying Proposition 4.11, we get that $\mathbf{A}$ is peculiar.

\section{Bipartite pseudo-BL algebras}

In this section, we shall define (strongly) bipartite pseudo-BL algebra and we shall prove some properties of them, following $[17,8]$.

A pseudo-BL algebra $\mathbf{A}$ is called bipartite if $U \cup U_{\sim}^{*}=U \cup U_{-}^{*}=A$ for some ultrafilter $U$ of A. A is called strongly bipartite if $U \cup U_{\sim}^{*}=U \cup U_{-}^{*}=A$ for any ultrafilter $U$ of A. Obviously, any strongly bipartite pseudo-BL algebra is bipartite.

A filter $F$ of $\mathbf{A}$ is called Boolean if for all $a \in A, a \vee a^{\sim} \in F$ and $a \vee a^{-} \in F$. It is obvious that if $F \subseteq G$ are two filters of $\mathbf{A}$ and $F$ is Boolean, then $G$ is also Boolean.

PROPOSITION 5.1. Let $\mathbf{A}$ be a pseudo-BL algebra and $F$ be a filter of $\mathrm{A}$. The following are equivalent:

(i) F is a Boolean ultrafilter of $\mathbf{A}$;

(ii) $F$ is a Boolean prime filter of $\mathbf{A}$;

(iii) $F$ is proper and for all $a \in A, a \in F$ or $\left(a^{\sim} \in F\right.$ and $\left.a^{-} \in F\right)$.

ProOF. (i) implies (ii). It is obvious, since, by Proposition 1.3, any ultrafilter of $\mathbf{A}$ is a prime filter of $\mathbf{A}$.

(ii) implies (iii). Let $a \in A$. Since $F$ is Boolean, we have that $a \vee a^{\sim} \in F$ and $a \vee a^{-} \in F$. Apply now the fact that $F$ is prime to get (iii).

(iii) implies (ii). Let $G$ be a proper filter of $A$ such that $F \subseteq G$ and suppose that $F \neq G$. Then there is $a \in G$ such that $a \notin F$. By (iii), it follows that $a^{\sim}, a^{-} \in F \subseteq G$, so by (8), $0=a^{\sim} \odot a \in G$, hence $G$ is not proper, that is a contradiction. Hence, $G=F$. Thus, $F$ is an ultrafilter of $\mathbf{A}$. Let us prove now that $F$ is Boolean. Let $a \in A$. If $a \in F$, since $a \leq a \vee a^{\sim}$ and $a \leq a \vee a^{-}$, we get that $a \vee a^{\sim}, a \vee a^{-} \in F$. If $a \notin F$, then $a^{\sim}, a^{-} \in F$ and from $a^{\sim} \leq a \vee a^{\sim}, a^{-} \leq a \vee a^{-}$we also get that $a \vee a^{\sim}, a \vee a^{-} \in F$.

LEMMA 5.2. Let $\mathbf{A}$ be a pseudo-BL algebra and $U$ be an ultrafilter of $\mathbf{A}$. The following are equivalent:

(i) $U \cup U_{\sim}^{*}=U \cup U_{-}^{*}=A$;

(ii) $U$ is Boolean.

Proof. Applying Proposition 5.1 (iii) and Remark 1.13 (ii) and (ii'), we get that $U$ is Boolean if and only if for all $a \in A, a \in U$ or ( $a^{\sim} \in U$ and $a^{-} \in U$ ) if and only if for all $a \in A, a \in U$ or $\left(a \in U_{\sim}^{*}\right.$ and $\left.a \in U_{-}^{*}\right)$ if and only if $U \cup U_{\sim}^{*}=U \cup U_{-}^{*}=A$. 
PROPOSITION 5.3. Let $\mathbf{A}$ be a pseudo-BL algebra $\mathbf{A}$. The following are equivalent:

(i) A is bipartite;

(ii) A has a Boolean proper filter.

ProOF. (i) implies (ii). Apply the above lemma.

(ii) implies (i). Suppose that $\mathbf{A}$ has a Boolean proper filter $F$. By Proposition 1.4, we can extend $F$ to an ultrafilter $U$ and $U$ is also Boolean. Applying again Lemma 5.2, we get that $\mathbf{A}$ is bipartite.

Let $\mathbf{A}$ be a pseudo-BL algebra. Following [17], we define

$$
\mathscr{B}(A)=\cap\{F \mid F \text { is a Boolean filter of } \mathbf{A}\},
$$

and

$$
\sup (A)=\left\{a \vee a^{\sim} \mid a \in A\right\} \cup\left\{a \vee a^{-} \mid a \in A\right\} .
$$

The following remark is obvious.

REMARK 5.4. Let $\mathbf{A}$ be a pseudo-BL algebra. Then

(i) $\mathscr{B}(A)$ is the smallest Boolean filter of $\mathbf{A}$;

(ii) if $\sup (A)$ is a filter of $\mathbf{A}$, then it is a Boolean filter;

(iii) $\sup (A) \subseteq \mathscr{B}(A)$.

PROPOSITION 5.5. Let $\mathrm{A}$ be a pseudo-BL algebra. Then

(i) $\mathscr{B}(A)=\langle\sup (A)\rangle$;

(ii) $\sup (A)=\left\{a \in A \mid a \geq a^{\sim}\right.$ or $\left.a \geq a^{-}\right\}$.

ProOF. (i) By the above remark, we have that $\sup (A) \subseteq \mathscr{B}(A)$ and $\mathscr{B}(A)$ is a filter of $\mathbf{A}$. Hence, $\langle\sup (A)\rangle \subseteq \mathscr{B}(A)$. Obviously, $\langle\sup (A)\rangle$ is a Boolean filter of $\mathbf{A}$, so $\mathscr{B}(A) \subseteq\langle\sup (A)\rangle$.

(ii) Let $a \in \sup (A)$. If $a=x \vee x^{\sim}$ for some $x \in A$ then, by (18), $a=x \vee x^{\sim} \geq$ $x^{\sim} \geq x^{\sim} \wedge x^{\approx}=\left(x \vee x^{\sim}\right)^{\sim}=a^{\sim}$. We prove similarly that if $a=x \vee x^{-}$for some $x \in A$, then $a \geq a^{-}$. Conversely, if $a \in A$ such that $a \geq a^{\sim}$, then $a=a \vee a^{\sim}$, hence $a \in \sup (A)$. Similarly, if $a \geq a^{-}$, then $a=a \vee a^{-}$, that is, $a \in \sup (A)$.

Proposition 5.6. Let $\mathbf{A}$ be a pseudo-BL algebra $\mathbf{A}$. The following are equivalent:

(i) A is strongly bipartite;

(ii) any ultrafilter of $\mathbf{A}$ is Boolean;

(iii) $\mathscr{B}(A) \subseteq \mathscr{M}(A)$, where we remind that $\mathscr{M}(A)$ denotes the intersection of all ultrafilters of $\mathbf{A}$. 
PROOF. (i) if and only if (ii). Apply Lemma 5.2.

(ii) implies (iii). If $U$ is an ultrafilter of $\mathbf{A}$ then, by (ii), $U$ is Boolean. Applying Remark 5.4 (i), we get that $\mathscr{B}(A) \subseteq U$.

(iii) implies (ii). Let $U$ be an ultrafilter of $\mathbf{A}$. Then $\mathscr{B}(A) \subseteq U$ and $\mathscr{B}(A)$ is a Boolean filter of $\mathbf{A}$. It follows that $U$ is also Boolean.

\section{References}

[1] R. Ambrosio and A. Lettieri, 'A classification of bipartite MV-algebras', Math. Japon. 38 (1993), 111-117.

[2] L. P. Belluce, A. Di Nola and A. Lettieri, 'Local MV-algebras', Rend. Circ. Mat. Palermo (2) 42 (1993), 347-361 (1994).

[3] R. Cignoli, I. M. L. D'Ottaviano and D. Mundici, Algebraic foundations of many-valued reasoning (Kluwer Acad. Publ., Dordrecht, 1998).

[4] A. Di Nola, G. Georgescu and A. Iorgulescu, 'Pseudo-BL algebras: Part I', Mult.-Valued Log., to appear.

[5] —, 'Pseudo-BL algebras: Part II', Mult.-Valued Log., to appear.

[6] A. Di Nola and A. Lettieri, 'Perfect MV-algebras are categorically equivalent to abelian $l$-groups', Studia Logica 53 (1994), 417-432.

[7] A. Di Nola, F. Liguori and S. Sessa, 'Using maximal ideals in the classification of MV-algebras', Portugal. Math. 50 (1993), 87-102.

[8] A. Di Nola, S. Sessa, F. Esteva, L. Godo and P. Garcia, 'The variety generated from perfect BL-algebras: an algebraic approach in fuzzy logic setting', preprint, 2000.

[9] A. Dvurecenskij, 'Pseudo MV-algebras are intervals in $\ell$-groups', J. Aust. Math. Soc. 72 (2002), 427-445.

[10] P. Flondor, G. Georgescu and A. Iorgulescu, 'Pseudo-t-norms and pseudo-BL algebras', Soft Computing 5 (2001), 355-371.

[11] G. Georgescu and A. Iorgulescu, 'Pseudo-MV algebras: a noncommutative extension of MValgebras', in: Information technology (Bucharest, 1999) (Inforec, Bucharest, 1999) pp. 961-968.

[12] - 'Pseudo-MV algebras', Mult.-Valued Log. 6 (2001), 95-135.

[13] — 'Pseudo-BL algebras: a noncommutative extension of BL-algebras (Abstract)', in: The Fifth International Conference FSTA 2000 on Fuzzy Sets Theory and its Application, February 2000 , pp. 90-92.

[14] P. Hájek, Metamathematics of fuzzy logic (Kluwer Acad. Publ., Dordrecht, 1998).

[15] 1. Leuştean, 'Local pseudo MV-algebras', Soft Computing 5 (2001), 386-395.

[16] D. Mundici, 'Interpretation of $A F C^{*}$-algebras in Lukasiewicz sentential calculus', J. Funct. Anal. 65 (1986), 15-63.

[17] E. Turunen, 'Boolean deductive systems of BL-algebras', Arch. Math. Logic 40 (2001), 467-473.

[18] - Mathematics behind fuzzy logic (Physica-Verlag, Heidelberg, 1999).

[19] E. Turunen and S. Sessa, 'Local BL-algebras', Mult.-Valued Log. 6 (2001), 1-21.

Faculty of Mathematics

University of Bucharest

14 Academiei Street

70109 Bucharest

Romania

e-mail: georgescu@funinf.math.unibuc.ro
National Institute for Research and Development in Informatics

8-10 Averescu Avenue

71316 Bucharest

Romania

e-mail: leo@u3.ici.ro 
